Pacific Journal of Mathematics

THE OSCULATORY BEHAVIOR OF SURFACES IN $\mathrm{P}^{5}$ 


\title{
THE OSCULATORY BEHAVIOR OF SURFACES IN $\mathbf{P}^{5}$
}

\author{
THEODORE SHIFRIN
}

\begin{abstract}
This article focuses on a certain class of surfaces in $\mathbf{P}^{5}$, exploiting the interplay between local projective differential geometry and global algebraic geometry. These are the so-called hypo-osculating surfaces, which have the property that at every point there is a hyperplane which is doubly tangent. The first main result of the paper, obtained by applying E. Cartan's method of moving frames, is that locally any such surface arises as a vector solution of one of the classical partial differential equations of physics (the wave equation or a generalized heat equation). Such equations were studied from a similar vantage point by G. Darboux and $C$. Segre, among others. The remainder of the paper is concerned with the global classification problem. Standard techniques in singularity theory yield formulas for the inflection cycles, and in the case of ruled surfaces there is an explicit numerical formula. By combining the earlier differential geometric results with Kodaira's classification of surfaces, one is able to arrive at a fairly complete understanding of the inflectionary behavior of hypo-osculating surfaces. In particular, the embedding of $\mathbf{P}^{1} \times \mathbf{P}^{1}$ as the quartic scroll is conjecturally the unique such smooth surface which is totally uninflected.
\end{abstract}

Introduction. In this paper we examine a class of surfaces in $\mathbf{P}^{5}$ which we call hypo-osculating. From the point of view of differential geometry, they arise as natural generalizations of classical developable surfaces in $\mathbf{R}^{3}$, i.e., flat surfaces. From the point of view of algebraic geometry, one seeks an algebraic surface $M$ and a very ample linear system (of dimension 5) on it with the property that through each point $p$ of $M$ passes an element of the linear system with at least a triple point at $p$; for then it follows that when we embed $M$ in $\mathbf{P}^{5}$ by the linear system, for each $p \in M$ there is a hyperplane $H$ in $\mathbf{P}^{5}$ which is "doubly tangent" to $M$ at $p$. That is, $H$ contains the osculating plane at $p$ of any curve in $M$ passing through $p$. Now it is natural to define the osculating space (or second-order tangent space) of $M$ at $p$ to be the linear span of the osculating planes of all curves in $M$ through $p$; ordinarily this is a five-dimensional space at most points of a general surface in $\mathbf{P}^{N}, N \geq 5$, but evidently for our special surface $M$ it can be everywhere at most four-dimensional. We call such a surface $M$ hypo-osculating; we call it perfectly hypo-osculating if its osculating space is exactly four-dimensional at every point. 
The study of the osculatory behavior of submanifolds has a venerable history, the Plücker formulas for algebraic curves (cf. [9]) being a prime example. C. Segre [18], and E. Togliatti [22] approached the question for surfaces by studying the partial differential equation (which Togliatti calls Laplace's equation) satisfied by the coordinates of the surface. In particular, let $(x, y) \in U \subset \mathbf{C}^{2}$ be local coordinates on $M \subset \mathbf{P}^{n}$ and $f: U \rightarrow$ $\mathrm{C}^{n+1}$ be a local holomorphic representation of $M$; then the osculating space is spanned by

$$
f, \quad \frac{\partial f}{\partial x}, \frac{\partial f}{\partial y}, \frac{\partial^{2} f}{\partial x^{2}}, \frac{\partial^{2} f}{\partial x \partial y}, \frac{\partial^{2} f}{\partial y^{2}},
$$

and $M$ 's being hypo-osculating corresponds to a differential equation

$$
a \frac{\partial^{2} f}{\partial x^{2}}+b \frac{\partial^{2} f}{\partial x \partial y}+c \frac{\partial^{2} f}{\partial y^{2}}+d \frac{\partial f}{\partial x}+e \frac{\partial f}{\partial y}+g f=0
$$

where $a, b, c, d, e$ and $g$ are holomorphic functions of $x, y$. Darboux [6] also studied surfaces in three-space satisfying such equations. Pohl in his thesis [16] defined higher order abstract osculating bundles in an attempt, among other things, to generalize the Plücker formulas to varieties of higher dimension. And studying the behavior of higher order osculating spaces has led recently to many beautiful results in theory of minimal surfaces (cf., for example, [5]), differential geometry/topology ([7]), and algebraic geometry ([10]).

We begin in $\$ 1$ with the requisite local differential geometry and some elementary results on the geometry of the Gauss map. We also discuss the relationship between the osculating spaces and the (projective) second fundamental form II, as in [10]. It then becomes clear that one needs a more subtle invariant than the rank of the Gauss map, namely the dimension of the linear system $|\mathrm{II}|$ at a generic point. In particular, hypo-osculating surfaces are characterized by the condition

$$
\operatorname{dim}\left|I_{x}\right|=1 \text {. }
$$

Following E. Cartan [2,3] we give in $\$ 2$ a local characterization of analytic (or, indeed $\mathscr{C}^{\infty}$ ) surfaces in $\mathbf{P}^{5}$ which are perfectly hypo-osculating:

(0.2) THEOREM. Let $M \subset \mathbf{P}^{5}$ have the property that its osculating spaces are everywhere four-dimensional. Then in a neighborhood of a generic point, $M$ is either

(i) of translation type

(ii) of "heat equation type"

or (iii) ruled. 
That is, in appropriate local coordinates, we can arrange that the partial differential equation (0.1) takes the forms

(i) $\frac{\partial^{2} f}{\partial x \partial y}=0$

(ii) $\frac{\partial^{2} f}{\partial y^{2}}=\varphi(x, y) \frac{\partial f}{\partial x}$

(iii) $\quad \frac{\partial^{2} f}{\partial y^{2}}=0$.

It is rather striking that the geometry of the problem leads to such a pretty reduction to the classical partial differential equations of physics. Note that from the point of view of differential equations case (i) is the generic one (i..e, the symbol is invertible); this is echoed by the algebro-geometric interpretation, as this is the case where the linear system $|\mathrm{II}|$ is base point free.

In $\$ 3$ we consider the global situation, discussing the osculating bundle and its associated Euler sequence. We give the Chern classes of the pertinent bundles, and then apply the Thom-Porteous theorem on singularities of bundle maps to compute the cycles of inflection points. Here, by inflection point we mean a point where the osculating space has less than generic dimension in $M$. In particular, one has the following

(0.3) Proposition. Let $M \subset \mathbf{P}^{5}$ be a smooth algebraic surface.

(a) If the osculating space of $M$ is generically of dimension 5 , then the one-cycle along which it drops rank is given by $6 H+4 K$, where $H$ is the hyperplane class and $K$ the canonical divisor.

(b) If $M$ is hypo-osculating and we assume that the line bundle generically defined by the differential equation (0.1) extends to a global line bundle $\mathscr{L}$ on $M$ (as in the case of ruled surfaces), then the locus of inflection points is a zero cycle of degree

(0.4) $\iota=11 K^{2}-5 c_{2}++21 d+28 K \cdot H+c_{1}(\mathscr{L}) \cdot(6 H+4 K)$

where $d=$ degree of $M, c_{2}=$ topological Euler characteristic.

As a consequence of (a) we deduce that the only smooth surface in $\mathbf{P}^{5}$ whose osculating space is everywhere five-dimensional is the Veronese embedding of $\mathbf{P}^{2}$ (this has been proved independently by Tai [21], and, in arbitrary dimension, by Fulton-Kleiman-Piene-Tai [8]). 
The remainder of the paper is devoted to specific applications of the numerical formula (0.4). In $\S 4$, we deal with ruled surfaces, proving that a ruled surface of genus $g$ and degree $d$ in $\mathbf{P}^{5}$ with finitely many inflection points has precisely $\iota=3(d+4(g-1))$ such. From this we deduce the

(0.5) THEOREM. There is a unique uninflected ruled surface in, $\mathbf{P}^{5}$, namely the image of $\mathbf{P}^{1} \times \mathbf{P}^{1}$ under the mapping

$$
(s, t) \mapsto\left(s, t, s^{2}, s t, s^{2} t\right) .
$$

Indeed, we offer the following

(0.6) Conjecture. The unique smooth perfectly hypo-osculating surface in $\mathbf{P}^{5}$ is the rational ruled surface above.

Even if we do not know in general whether $\mathscr{L}$ globalizes we can still use formula (0.4) to classify perfectly hypo-osculating surfaces. For in this case $\mathscr{L}$ is globally defined and, by definition, one must have no inflection points at all, i.e., $\iota=0$. The classification problem then reduces to relating the numerical characters of the surfaces.

In $\$ 5$ we begin with two global examples, one of wave equation type and one of heat equation type, essentially the two simplest possible. As evidence for the conjecture, we point out that in these cases either the surfaces inflects or it acquires singularities. Now, how might a non-ruled surface be perfectly hypo-osculating? It might be globally of wave or heat equation type, or it might be generically of wave equation type, but of heat equation type along a subvariety. The latter case is particularly troublesome, and we can deal with it only when the degeneration involves the surface's acquiring lines. In this section, regardless, we compute the number (0.4) of inflection points for the various surfaces on Kodaira's list ([13], [9]). Using an assortment of methods, we are able to provide good evidence for the conjecture, but there are some cases which remain unresolved here.

The author would like to thank Michael Artin for suggesting the problem to him, and Phillip Griffiths, Robert Bryant, Woody Lichtenstein, Clint McCrory, and particularly Robert Varley for helpful conversations.

1. Projective geometry: the Gauss map and second fundamental form. Let $M \subset \mathbf{P}^{n}$ be a smooth surface, and let $\pi: \mathbf{C}^{n+1}-\{0\} \rightarrow \mathbf{P}^{n}$ be the canonical projection. We define the frame bundle $\mathscr{F}(M) \rightarrow M$ as the fiber bundle whose fiber over $z \in M$ consists of all bases $Z_{0}, \ldots, Z_{n}$ for 
$\mathbf{C}^{n+1}$ satisfying

(i) $\pi\left(Z_{0}\right)=z$

(ii) $Z_{0} \wedge Z_{1} \wedge Z_{2}$ is the $\mathbf{P}^{2} \subset \mathbf{P}^{n}$ tangent to $M$ at $z$;

we write the latter tangent space as $\mathbf{P} \tilde{T}_{z} M$, letting $\tilde{T}_{z} M=\operatorname{span}\left(Z_{0}, Z_{1}, Z_{2}\right)$ represent its lift to $\mathbf{C}^{n+1}$. This frame bundle evidently has as structure group $G \subset \mathrm{Gl}(n+1, \mathrm{C})$ all matrices of the form

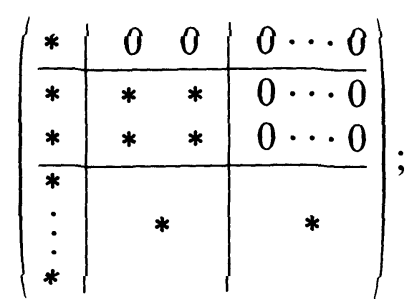

we refer to a local section of $\mathscr{F}(M)$ as a (local) adapted frame field on $M$; note this makes sense in both the smooth and the analytic categories.

Using $0 \leq i, j, k \leq n, 1 \leq \alpha, \beta, \gamma \leq 2$, and $3 \leq \mu, \nu \leq n$, we define 1 -forms $\omega_{j}^{j}$ on $\mathscr{F}(M)$ by viewing $Z_{i}$ as the obvious vector-valued function on $\mathscr{F}(M)$ and setting

$$
d Z_{i}=\sum \omega_{i}^{j} Z_{j} .
$$

We write $\omega_{0}^{j}=\omega^{j}$ and observe that property (ii) above implies that $\omega^{\mu}=0$. Differentiating (1.1) gives the crucial structure equations

$$
d \omega_{i}^{j}=\sum \omega_{i}^{k} \wedge \omega_{k}^{J}
$$

in particular, differentiating $\omega^{\mu}=0$ and applying the Cartan lemma, we infer that

$$
\omega_{\alpha}^{\mu}=\sum h_{\alpha \beta}^{\mu} \omega^{\beta}, \quad h_{\alpha \beta}^{\mu}=h_{\beta \alpha}^{\mu} .
$$

Following classical differential geometry, define the (projective) second fundamental form of $M$ by

$$
\mathrm{II}=\sum h_{\alpha \beta}^{\mu} \omega^{\alpha} \otimes \omega^{\beta} \otimes Z_{\mu}
$$

considered with values in $\mathbf{C}^{n+1} / \tilde{T} M$. We claim II is an intrinsically defined quadratic form on $M$ with values in the normal bundle $N\left(M, \mathbf{P}^{n}\right)$ of $M$.

For let $a=\left(a_{i j}\right)$ be a local map from $M$ to $G$; then

$$
\begin{aligned}
& Z_{0}^{*}=a_{0}^{0} Z_{0} \\
& Z_{\alpha}^{*}=a_{\alpha}^{0} Z_{0}+\sum a_{\alpha}^{\beta} Z_{\beta} \\
& Z_{\mu}^{*}=a_{\mu}^{0} Z_{0}+\sum a_{\mu}^{\beta} Z_{\beta}+\sum a_{\mu}^{\nu} Z_{\nu} .
\end{aligned}
$$


Put $\omega=\left(\omega_{l}^{j}\right)$; then we have the transformation rule $\omega^{*}=a \omega a^{-1}+$ $d a \cdot a^{-1}$. Therefore, computing the effect on II,

$$
\begin{aligned}
\sum \omega_{\alpha}^{\mu *} \otimes \omega^{\alpha *} \otimes Z_{\mu}^{*} & =\sum a_{\alpha}^{i} \omega_{i}^{j}\left(a^{-1}\right)_{j}^{\mu} \otimes a_{0}^{k} \omega_{k}^{l}\left(a^{-1}\right)_{l}^{\alpha} \otimes a_{\mu}^{m} Z_{m} \\
& =a_{0}^{0} \sum \omega_{a}^{j} \otimes \omega^{\alpha} \otimes Z_{j} \\
& =a_{0}^{0} \sum \omega_{\alpha}^{\mu} \otimes \omega^{\alpha} \otimes Z_{\mu} \bmod \left(Z_{0}, Z_{1}, Z_{2}\right) ;
\end{aligned}
$$

since $Z_{0}$ is a local section of the tautological bundle $\mathcal{O}_{M}(-1)$, we infer that this is indeed a well-defined section of $\left(\mathbf{C}^{n+1} / \tilde{T} M\right) \otimes \mathcal{O}_{M}(1)$. The Euler sequence ([19]) gives

$$
\left\{\begin{array}{l}
0 \rightarrow \mathcal{O}(-1) \rightarrow \mathbf{C}^{n+1} \rightarrow T \mathbf{P}^{n} \otimes \mathcal{O}(-1) \rightarrow 0 \\
0 \rightarrow \mathcal{O}_{M}(-1) \rightarrow \tilde{T} M \rightarrow T M \otimes \mathcal{O}_{M}(-1) \rightarrow 0,
\end{array}\right.
$$

whence $N\left(M, \mathbf{P}^{n}\right)=T \mathbf{P}^{n} / T M \cong\left(\mathbf{C}^{n+1} / \tilde{T} M\right) \otimes \mathcal{O}_{M}(1)$, and so II is indeed a well-defined map $\operatorname{Sym}^{2}(T M) \rightarrow N\left(M, \mathbf{P}^{n}\right)$.

We observe that $\mathrm{II}^{\mu}=\sum h_{\alpha \beta}^{\mu} \omega^{\alpha} \otimes \omega^{\beta}, \mu=3, \ldots, n$, give a family of quadratic forms on $T_{z} M$ parametrized by $N_{z}^{*} M$, hence a linear system of conics on $\mathbf{P}\left(T_{z} M\right) \cong \mathbf{P}^{1}$. Since the complete linear system of conics on $\mathbf{P}^{1}$ is (projectively) two-dimensional, the linear subsystem $|\mathrm{II}|$ spanned by $\left\{\mathrm{II}^{\mu}\right\}$ has dimension as most two; so by a change of basis in $N_{z} M$, we may assume that $\omega_{\alpha}^{\mu}=0$ for $\mu>3+\operatorname{dim}|\mathrm{II}|$. Letting $3 \leq \rho, \sigma \leq 3+$ $\operatorname{dim}|\mathrm{II}|$, we see that $d Z_{\alpha} \equiv 0 \bmod \left(Z_{0}, Z_{\beta}, Z_{\rho}\right)$, and we set $\tilde{\mathscr{T}}_{z}^{(2)} M=$ $\operatorname{span}\left\{Z_{0}, Z_{\alpha}, Z_{\rho}\right\}$. Note that $\mathbf{P}\left(\tilde{\mathscr{T}}_{z}^{(2)} M\right)$, the projective second-order tangent space of $M$ at $z$, contains the osculating planes at $z$ of all curves on $M$ passing through $z$. For let $C=\{Z(t)\}$ be such a curve with $Z(0)=z$, and let $Z_{0}(t), Z_{1}(t), \ldots, Z_{n}(t)$ be a section of $\mathscr{F}(M)$ over $C$ with $Z_{1}(t)=Z_{0}^{\prime}(t)$. By definition, the osculating plane of $C$ at $z$ is $Z_{0}(0) \wedge$ $Z_{0}^{\prime}(0) \wedge Z_{0}^{\prime \prime}(0)$, and $Z_{0}^{\prime \prime}(0)=\left(d Z_{1} / d t\right)(0) \in \tilde{\mathscr{T}}_{z}^{(2)} M$. Note it follows immediately from our definition that the projective second-order tangent or osculating space $\mathbf{P}\left(\tilde{\mathscr{T}}_{z}^{(2)} M\right)$ has dimension $3+\operatorname{dim}\left|I_{z}\right|$.

Now, if $\operatorname{dim} \mathbf{P}\left(\tilde{\mathscr{T}}^{(2)} M\right)<n$, then we may analogously define the third fundamental form III: $\operatorname{Sym}^{3}(T M) \rightarrow\left(\mathbf{C}^{n+1} / \tilde{T}^{(2)} M\right) \otimes \mathcal{O}_{M}(1)$ by differentiating the equations $\omega_{\alpha}^{\tau}=0, \tau>\operatorname{dim}\left(\mathbf{P} \tilde{\mathscr{T}}^{(2)} M\right)$ :

$$
\text { III }=\sum \omega_{\rho}^{\tau} \otimes \omega_{\alpha}^{\rho} \otimes \omega^{\alpha} \otimes Z_{\tau} .
$$

Since we will need III only briefly later in our work, we refer the reader to [10] for details.

Classically, surfaces in $\mathbf{R}^{3}$ with zero Gaussian curvature were characterized (at least locally) as ruled surfaces whose tangent plane is constant along rulings; such surfaces were called developable. Interpreting Gaussian curvature as the Jacobian of the Gauss map $\gamma: M \rightarrow S^{2}$, we 
find that the same result holds projectively, and in higher codimension as well.

Let $M^{2} \subset \mathbf{P}^{n}$ be smooth. Let $\mathbf{G}(2, n)$ be the Grassmannian of $\mathbf{P}^{2}$ 's in $\mathbf{P}^{n}$ (which is naturally $G(3, n+1)$ ), and define the (projective) Gauss mapping $\gamma: M \rightarrow \mathbf{G}(2, n)$ by $\gamma(z)=\tilde{T}_{z} M$. Then we have the following

(1.6) Proposition. Let $M^{2} \subset \mathbf{P}^{n}$ and suppose the Gauss map $\gamma$ has rank one. Then $M$ is developable.

Proof. Choose an adapted frame field $Z_{0}, Z_{1}, \ldots, Z_{n}$ so that $\pi_{*} Z_{1} \in$ ker $d \gamma$. Since $\gamma(z)=Z_{0} \wedge Z_{1} \wedge Z_{2}$, we find that

$$
d \gamma \equiv \sum \omega_{1}^{\mu} Z_{0} \wedge Z_{\mu} \wedge Z_{2}+\omega_{2}^{\mu} Z_{0} \wedge Z_{1} \wedge Z_{\mu}(\bmod \gamma)
$$

Therefore $\omega_{\alpha}^{\mu} \equiv 0 \bmod \left(\omega^{2}\right)$. From the symmetry (1.3) of the second fundamental form, we infer that $\omega_{1}^{\mu}=0$ and $\omega_{2}^{\mu}=h_{\mu} \omega^{2}$. Since $0=\omega_{1}^{\mu}=\omega_{1}^{2} \wedge$ $\omega_{2}^{\mu}$, it follows that $\omega_{1}^{2} \equiv 0 \bmod \left(\omega^{2}\right)$, and thence that the line $Z_{0} \wedge Z_{1}$ is ruling of $M$, for

$$
\begin{aligned}
d\left(Z_{0} \wedge Z_{1}\right) & =\omega^{2} Z_{2} \wedge Z_{1}+\omega_{1}^{2} Z_{0} \wedge Z_{2}+\sum \omega_{1}^{\mu} Z_{0} \wedge Z_{\mu} \\
& \equiv 0 \bmod \left(\omega^{2}\right) .
\end{aligned}
$$

It now becomes clear that for surfaces in $\mathbf{P}^{n}, n \geq 4$, we need something more refined than the rank of the Gauss map. Since the derivative of the Gauss map, as we saw in (1.7), is captured by the second fundamental form, it is natural to look at the next simplest numerical invariant - the dimension of the linear system $|\mathrm{II}|$ at a general point of $M$. As we pointed out earlier, $\operatorname{dim}|I I| \leq 2$, and so there are four possibilities. The generic case, of course, is that $\operatorname{dim}|\mathrm{II}|=2$, and here there's nothing to say. If $\operatorname{dim}|\mathrm{II}|<2$, we say $M$ is hypo-osculating, and if, moreover, it is constant on $M$, we say $M$ is perfectly hypo-osculating.

Case (i). $\operatorname{dim}|\mathrm{II}|=-1$, i.e., $|\mathrm{II}|=\varnothing$; so $\omega_{\alpha}^{\mu}=0$ for all $\alpha$, $\mu$. Therefore the tangent plane to $M$ is constant, and $M$ is a subset of a plane.

Case (ii). $\operatorname{dim}|\mathrm{II}|=0$. Choose $Z_{3}$ spanning $\tilde{\mathscr{T}}_{z}^{(2)} / \tilde{T}_{z}$, and then $\mathrm{II}=$ $\sum h_{\alpha \beta}^{3} \omega^{\alpha} \otimes \omega^{\beta} \otimes Z_{3}$. Now the quadratic form $\left(h_{\alpha \beta}^{3}\right)$ has rank either 1 or 2 . In the former case, we may take $h_{11}^{3}=h_{12}^{3}=0, h_{22}^{3}=1$, and II $=\omega^{2} \otimes \omega^{2}$ $\otimes Z_{3}$; this is the second fundamental form of a developable surface (cf. the proof of (1.5)). In the latter case, we may take $h_{11}^{3}=h_{22}^{3}=0, h_{12}^{3}=1$, and so $\omega_{1}^{3}=\omega^{2}, \omega_{2}^{3}=\omega^{1}, \omega_{\alpha}^{\sigma}=0$ for $\sigma \geq 4$. Using the structure equation, we find $0=d \omega_{\alpha}^{\sigma}=\omega_{\alpha}^{3} \wedge \omega_{3}^{\sigma}$; therefore, $\omega_{3}^{\sigma} \wedge \omega^{1}=\omega_{3}^{\sigma} \wedge \omega^{2}=0$, and so 
$\omega_{3}^{\sigma}=0$. We conclude that the osculating space $\tilde{\mathscr{T}}_{z}^{(2)} M=Z_{0} \wedge Z_{1} \wedge Z_{2} \wedge$ $Z_{3}$ is constant, from which it follows that $M \subset \mathbf{P}^{3}$.

Case (iii). $\operatorname{dim}|\mathrm{II}|=1$. This is the interesting case to which this paper is devoted. As examples of this phenomenon we have non-developable ruled surfaces (here $\left.|I I|=\left|\left(\omega^{1}\right)^{2}, \omega^{1} \omega^{2}\right|\right)$ and surfaces of translation type (here $\left.|I I|=\left|\left(\omega^{1}\right)^{2},\left(\omega^{2}\right)^{2}\right|\right)$. However, there are yet others, and it is to the local classification problem that we turn next.

2. Local theory. We are seeking a (local) characterization of surfaces $M$ in $\mathbf{P}^{5}$ with the property that $\operatorname{dim} \mathbf{P} \tilde{T}_{z}^{(2)} M=4$ for generic $z \in M$. From an analytic point of view, represent a local lifting of surface as the image of a function $f: U \subset \mathbf{C}^{2} \rightarrow \mathbf{C}^{6}$; then there are functions $a, b, c, d, e, g$ with

$$
a \frac{\partial^{2} f}{\partial x^{2}}+b \frac{\partial^{2} f}{\partial x \partial y}+c \frac{\partial^{2} f}{\partial y^{2}}+d \frac{\partial f}{\partial x}+e \frac{\partial f}{\partial y}+g f=0
$$

(and no further relations should exist). One might then hope to find a local analytic change of coordinates transforming (2.1) to one of the following forms:

$$
\begin{aligned}
& \text { wave equation: } \frac{\partial^{2} f}{\partial x \partial y}=0 \\
& \text { heat equation: } \frac{\partial^{2} f}{\partial y^{2}}=\varphi(x, y) \frac{\partial f}{\partial x} \\
& \text { degenerate heat equation: } \frac{\partial^{2} f}{\partial y^{2}}=0 .
\end{aligned}
$$

Our goal in this section is to obtain such a local classification by using the methods of Elie Cartan (cf. [2], [3], [4]).

We begin with an elementary

(2.2) Lemma. After a change of coordinates, a pencil of conics on $\mathbf{P}^{1}$ is either of the form $\left|x^{2}, x y\right|$ (a pencil with base point) or of the form $\left|x^{2}, y^{2}\right|$ (base point free).

Proof. If the linear system has a base point, by a change of coordinates on $\mathbf{P}^{1}$ we may assume that it is $x=0$. Then clearly $x^{2}, x y$ are a basis for the subspace of quadratic polynomials which are divisible by $x$. On the other hand, if the linear system has no base point, then consider 
the 2-to-1 map $\mathbf{P}^{1} \rightarrow \mathbf{P}^{1}$ given by the linear system; by the RiemannHurwitz formula, it has two branch points, which we may take to be 0 and $\infty$.

We begin our study with the case that $|\mathrm{II}|$ has a base point, i.e., we assume $|\mathrm{II}|$ is given by $\left|\left(\omega^{1}\right)^{2}, \omega^{1} \omega^{2}\right|$, or, more specifically that II $=\left(\omega^{1}\right)^{2} \otimes$ $Z_{3}+2 \omega^{1} \omega^{2} \otimes Z_{4}$. This limits us to frame fields $Z_{0}, Z_{1}, \ldots, Z_{5}$ with

$$
\begin{array}{lll}
\omega_{1}^{3}=\omega^{1}, & \omega_{1}^{4}=\omega^{2}, & \omega_{1}^{5}=0 \\
\omega_{2}^{3}=0, & \omega_{2}^{4}=\omega^{1}, & \omega_{2}^{5}=0
\end{array} .
$$

More precisely, we work on the integral submanifolds of (2.3) in $\mathscr{F}(M)$. Soon we shall cut these down even further.

Differentiating $\omega_{2}^{3}=0$ gives

$$
\begin{aligned}
0 & =d \omega_{2}^{3}=\omega_{2}^{1} \wedge \omega_{1}^{3}+\omega_{2}^{2} \wedge \omega_{2}^{3}+\omega_{2}^{3} \wedge \omega_{3}^{3}+\omega_{2}^{4} \wedge \omega_{4}^{3} \\
& =\left(\omega_{2}^{1}-\omega_{4}^{3}\right) \wedge \omega^{1}
\end{aligned}
$$

and so we deduce that

$$
\omega_{2}^{1}-\omega_{4}^{3} \equiv 0 \quad \bmod \left(\omega^{1}\right) .
$$

Similarly, differentiating $\omega_{2}^{4}=\omega^{2}$ gives

$$
\begin{aligned}
\omega_{2}^{1} \wedge \omega_{1}^{4}+\omega_{2}^{2} \wedge \omega_{2}^{4}+\omega_{2}^{3} \wedge \omega_{3}^{4}+\omega_{2}^{4} \wedge \omega_{4}^{4} \\
=\omega_{0}^{0} \wedge \omega^{1}+\omega^{1} \wedge \omega_{1}^{1}+\omega^{2} \wedge \omega_{2}^{1}, \\
\left(\omega_{2}^{2}-\omega_{4}^{4}-\omega_{0}^{0}+\omega_{1}^{1}\right) \wedge \omega^{1}+\left(2 \omega_{2}^{1}\right) \wedge \omega^{2}=0 .
\end{aligned}
$$

Therefore, by the Cartan lemma, there are functions $a, b, c$ on $\mathscr{F}(M)$ such that

$$
-\omega_{0}^{0}+\omega_{1}^{1}+\omega_{2}^{2}-\omega_{4}^{4}=a \omega^{1}+2 b \omega^{2}, \quad \omega_{2}^{1}=b \omega^{2}+c \omega^{2} .
$$

Differentiating the latter equation in (2.5), we find

$$
\begin{aligned}
\omega_{2}^{0} \wedge \omega^{1}+\left(\omega_{2}^{2}-\omega_{1}^{1}\right) \wedge \omega_{2}^{1}= & \left(d b+b\left(\omega_{0}^{0}-\omega_{1}^{1}\right)-c \omega_{1}^{2}\right) \wedge \omega^{1} \\
& +\left(d c-b \omega_{2}^{1}+c\left(\omega_{0}^{0}-\omega_{2}^{2}\right)\right) \wedge \omega^{2} .
\end{aligned}
$$

Therefore

$$
\begin{array}{ll}
\omega_{2}^{0}+b\left(\omega_{2}^{2}-\omega_{1}^{1}\right)-d b+b\left(\omega_{1}^{1}-\omega_{0}^{0}\right)+c \omega_{1}^{2} \equiv 0 & \bmod \left(\omega^{1}, \omega^{2}\right) \\
c\left(\omega_{2}^{2}-\omega_{1}^{1}\right)-d c+c\left(\omega_{2}^{2}-\omega_{0}^{0}\right) \equiv 0 &
\end{array}
$$

That is,

$$
\begin{aligned}
& d c+c\left(\omega_{0}^{0}+\omega_{1}^{1}-2 \omega_{2}^{2}\right) \equiv 0 \\
& d b+b\left(\omega_{0}^{0}-\omega_{2}^{2}\right)-\left(c \omega_{1}^{2}+\omega_{2}^{0}\right) \equiv 0
\end{aligned}
$$


At this point, there are two possibilities: (i) the function $c$ is identically zero, or (ii) the function $c$ is never zero (locally on $M$ ).

First we dispense with the first case. If $c=0$, then, $\omega_{2}^{1} \equiv 0 \bmod \left(\omega^{1}\right)$, and this implies the surface is ruled, for from

$$
\begin{aligned}
d\left(Z_{0} \wedge Z_{2}\right) \equiv & \omega^{1} Z_{1} \wedge Z_{2}+\omega_{2}^{1} Z_{0} \wedge Z_{1}+\omega_{2}^{3} Z_{0} \wedge Z_{3} \\
& +\omega_{2}^{4} Z_{0} \wedge Z_{4} \bmod \left(Z_{0} \wedge Z_{2}\right)
\end{aligned}
$$

and (2.3) we infer that $Z_{0} \wedge Z_{2}$ is constant along integral curves of $Z_{2}$; i.e., the integral curves are lines.

In the second, and more interesting, case, we claim that we can refine our frame so as to have $\omega_{2}^{1}=\omega^{2}$ (i.e., $b=0, c=1$ ). This follows immediately from (2.6). For example, at a fixed point $z$ in $M$, note that replacing $Z_{1}$ by $\gamma Z_{1}$ and keeping the other vectors fixed results in the transformation $\omega_{2}^{1} \rightarrow(1 / \gamma) \omega_{2}^{1}$, and so with this 1-parameter group we can choose a frame at $z$ with $c=1$. Now by moving along an integral manifold of $\omega_{0}^{0}+\omega_{1}^{1}-2 \omega_{2}^{2}=0$, we guarantee $c \equiv 1$. The case for the function $b$ is treated similarly, noting here that the 1-parameter group acts by affine transformations. More specifically, consider the transformation $Z_{2} \rightarrow t Z_{0}+Z_{2}$, fixing $Z_{0}, Z_{1}, Z_{\mu}$. Then this results in $\omega_{2}^{0} \rightarrow \omega_{2}^{0}+d t$, and the second equation in (2.6) reduces to $d b-d t=0$. By solving, we clearly can arrange for a frame so that $b=0$ at $z$; now move along integral manifolds of $\omega_{0}^{0}-\omega_{2}^{2}=0, \omega_{1}^{2}+\omega_{2}^{0}=0$ (inside the integral manifolds of (2.3), of course), and we obtain $b=0$. One checks that we have imposed independent conditions on $\mathscr{F}(M)$; at this point there is no subtlety involved.

In order to pin down the geometry further, we examine the third fundamental form, i.e., the forms $\omega_{3}^{5}$ and $\omega_{4}^{5}$. From differentiating $\omega_{1}^{5}=$ $\omega_{2}^{5}=0$ we see

$$
\begin{aligned}
& \omega_{1}^{3} \wedge \omega_{3}^{5}+\omega_{1}^{4} \wedge \omega_{4}^{5}=0, \quad \text { i.e. } \\
& \omega_{3}^{5} \wedge \omega^{1}+\omega_{4}^{5} \wedge \omega^{2}=0
\end{aligned}
$$

and

$$
\begin{aligned}
& \omega_{2}^{3} \wedge \omega_{3}^{5}+\omega_{2}^{4} \wedge \omega_{4}^{5}=0, \quad \text { i.e. } \\
& \omega_{4}^{5} \wedge \omega^{1}=0 .
\end{aligned}
$$

From (2.7) we obtain the usual symmetry condition, which, together with (2.8), allows us to conclude that

$$
\begin{aligned}
& \omega_{3}^{5}=\alpha \omega^{1}+\beta \omega^{2} \\
& \omega_{4}^{5}=\beta \omega^{1} .
\end{aligned}
$$


Proceeding as above, we differentiate $\omega_{4}^{5}=\beta \omega^{1}$ to obtain

$$
d \beta+\beta\left(\omega_{0}^{0}-\omega_{1}^{1}-\omega_{4}^{4}+\omega_{5}^{5}\right) \equiv 0 \bmod \left(\omega^{1}\right) .
$$

Now either $\beta$ is identically zero, or, if $\omega_{0}^{0}-\omega_{1}^{1}-\omega_{4}^{4}+\omega_{5}^{5}$ is not already horizontal, we can, as above, choose a frame so as to make $\beta$ identically equal to 1 . From earlier refinements, $\omega_{0}^{0}=\omega_{1}^{1}$, but now setting $\beta=1$ forces $\omega_{4}^{4}=\omega_{5}^{5}$.

Keeping the two possible values of $\beta$ in mind, we next set to work on $\omega_{3}^{5}$. Differentiating $\omega_{3}^{5}=\alpha \omega^{1}+\beta \omega^{2}$, we obtain

$$
\begin{array}{ll} 
& d \alpha+\alpha\left(\omega_{0}^{0}-\omega_{1}^{1}-\omega_{3}^{3}+\omega_{5}^{5}\right)-\beta\left(\omega_{1}^{2}+\omega_{3}^{4}\right) \equiv 0 \bmod \left(\omega^{1}, \omega^{2}\right), \\
& d \alpha+\alpha\left(\omega_{3}^{5}-\omega_{3}^{3}\right)-\beta\left(\omega_{1}^{2}+\omega_{3}^{4}\right) \equiv 0 \bmod \left(\omega^{1}, \omega^{2}\right) .
\end{array}
$$

Case (i): $\beta=0$. If $\alpha=0$, then one checks easily that $M \subset \mathbf{P}^{4}$, in analogy with the case with $\operatorname{dim}|\mathrm{II}|=0$ discussed in $\$ 1$. If not, proceeding as before, we may arrange $\alpha=1$, and this results in the normalization

$$
\omega_{3}^{5}=\omega^{1}, \omega_{4}^{5}=0 \text {. }
$$

Note that in this case we have (cf. (1.5))

$$
|\mathrm{III}|=\left|\left(\omega^{1}\right)^{3}\right| \text {. }
$$

Case (ii): $\beta=1$. Then, assuming the form $\omega_{1}^{2}+\omega_{3}^{4}$ is not horizontal, we may refine the frames to obtain $\alpha=0$. However, there is something to check here. Differentiating the equation $\omega_{1}^{4}=\omega^{2}$ gives us the fact that $2 \omega_{1}^{2}-\omega_{3}^{4}$ is horizontal; moreover, we have imposed the equality $\omega_{2}^{0}+\omega_{1}^{2}$ $=0$. So, clearly, $\omega_{1}^{2}+\omega_{3}^{4}$ is horizontal if and only if $\omega_{2}^{0}$ if horizontal, and it clearly is not (cf. discussion of case (ii) following (2.6)). So now we obtain

$$
\omega_{3}^{5}=\omega^{2}, \quad \omega_{4}^{5}=\omega^{1}
$$

In this case, we have

$$
\mid \text { III }|=|\left(\omega^{1}\right)^{2} \omega^{2} \mid
$$

Regardless of the nature of their third fundamental forms, we now wish to characterize the surfaces with $|\mathrm{II}|=\left|\left(\omega^{1}\right)^{2}, \omega^{1} \omega^{2}\right|$.

(2.14) THEOREM. If $|\mathrm{II}|$ has a base point, then in a neighborhood of a generic point, either the surface is ruled or there are local coordinates $x, y$ and a local lifting $f: U \rightarrow \mathbf{C}^{6}-\{0\}$ of $M$ such that

$$
\frac{\partial^{2} f}{\partial y^{2}}=\varphi(x, y) \frac{\partial f}{\partial x}
$$

for some (analytic) function $\varphi$. 
Proof. We have seen that if $\omega_{2}^{1} \equiv 0 \bmod \left(\omega^{1}\right)$, then $M$ is ruled. If not, then we have the following:

$$
\begin{array}{llll} 
& \omega_{1}^{3}=\omega^{1} & \omega_{1}^{4}=\omega^{2} & \omega_{4}^{5}=0 \\
\omega_{2}^{1}=\omega^{2} & \omega_{2}^{3}=0 & \omega_{2}^{4}=\omega^{1} & \omega_{2}^{5}=0
\end{array}
$$

Fix now a particular frame field $Z_{0}, \ldots, Z_{5}$ with respect to which these formulas hold, and pick local coordinates $x, y$ on $M$ so that $\omega^{1}=$ $d x / p(x, y), \omega^{2}=d y / q(x, y), p, q \neq 0$. Then setting $Z_{0}=f(x, y)$, we have

$$
Z_{1}=p \frac{\partial f}{\partial x} \quad \text { and } \quad Z_{2}=q \frac{\partial f}{\partial y}
$$

and comparing coefficients of $\omega^{2}$ in the equation

$$
d Z_{2}=\sum \omega_{2}^{j} Z_{j} \equiv \omega^{2} Z_{1}+\omega^{1} Z_{4} \quad \bmod Z_{0}, Z_{2}
$$

we infer that

$$
q^{2} \frac{\partial^{2} f}{\partial y^{2}}+\left(q \frac{\partial f}{\partial y}+\cdots\right) \frac{\partial f}{\partial y}-p \frac{\partial f}{\partial x}+(\cdots) f=0 .
$$

(The $Z_{0}$ term could be eliminated a priori by further refining our frame field to annihiliate $\omega_{2}^{0}$.) It follows from an easy application of the Cauchy-Kowalewski Theorem that we can replace $f$ by $\psi(x, y) f$ to remove the $(\cdots) f$ term. Then an easy calculus exercise shows we can eliminate the $(\cdots) \partial f / \partial y$ term by a change of coordinates. This proves the theorem.

We now turn to the case that the linear system $|\mathrm{II}|$ has no base point. We may assume that $|\mathrm{II}|=\left|\left(\omega^{1}\right)^{2},\left(\omega^{2}\right)^{2}\right|$, and therefore take

$$
\begin{array}{lll}
\omega_{1}^{3}=\omega^{1}, & \omega_{1}^{4}=0, & \omega_{1}^{5}=0 \\
\omega_{2}^{3}=0, & \omega_{2}^{4}=\omega^{2}, & \omega_{2}^{5}=0 .
\end{array}
$$

Differentiating $\omega_{1}^{4}=0$ gives

$$
-\omega_{3}^{4} \wedge \omega^{1}+\omega_{1}^{2} \wedge \omega^{2}=0
$$

whence $\omega_{1}^{2}$ is horizontal; in a similar fashion we find the same is true of $\omega_{2}^{1}$. Letting $\omega_{1}^{2}=a \omega^{1}+b \omega^{2}$ and $\omega_{2}^{1}=\alpha \omega^{1}+\beta \omega^{2}$ and doing the same calculations as before, we obtain

$$
\begin{aligned}
& d a+a\left(\omega_{0}^{0}-2 \omega_{1}^{1}+\omega_{2}^{2}\right)+\omega_{3}^{2} \equiv 0 \\
& d b+b\left(\omega_{0}^{0}-\omega_{1}^{1}\right)-\omega_{1}^{0} \equiv 0 \\
& d \alpha+\alpha\left(\omega_{0}^{0}-\omega_{2}^{2}\right)-\omega_{2}^{0} \equiv 0 \\
& d \beta+\beta\left(\omega_{0}^{0}+\omega_{1}^{1}-2 \omega_{2}^{2}\right)+\omega_{4}^{1} \equiv 0
\end{aligned}
$$


It follows, as before, that we may choose frame fields so that $\omega_{1}^{2}=\omega_{2}^{1}=0$. Set $\omega^{1}=d x / p(x, y), \omega^{2}=d y / q(x, y), Z_{0}=(1, f(x, y))$ with $f: U \rightarrow \mathbf{C}^{5}$; then from

$$
\begin{array}{ll}
d Z_{1} \equiv \omega_{1}^{2} Z_{2}+\omega_{1}^{3} Z_{3}+\omega_{1}^{4} Z_{4} & \bmod Z_{1} \\
d Z_{2} \equiv \omega_{2}^{1} Z_{1}+\omega_{2}^{3} Z_{3}+\omega_{2}^{4} Z_{4} & \bmod Z_{2}
\end{array}
$$

it follows that $\partial^{2} f / \partial x \partial y \equiv 0 \bmod Z_{1}$ and $\bmod Z_{2}$, whence $\partial^{2} f / \partial x \partial y=0$. Thus the surface may be represented (in affine coordinates) locally by solutions of the wave equation.

We summarize the results in the following

(2.17) THEOREM. Let $M^{2} \subset \mathbf{P}^{5}$ be a hypo-osculating surface, i.e., $\operatorname{dim} \mathbf{P} \tilde{T}_{z}^{(2)} M \leq 4$ for all $z \in M$, which does not lie in a hyperplane. Then in a neighborhood of a generic point $M$ is either ruled (developable if $\operatorname{dim} \mathbf{P} \tilde{T}_{z}^{(2)} M=3$ ) or of heat or wave equation type.

REMARK. While our major interest is in the complex analytic case, this result is equally valid in the (real) smooth case. In fact, it is worth pointing out that a general minimal surface $M^{2} \subset S^{n}$ is of wave equation type. Recall (cf. [5]) that a minimal surface is defined by the condition that $\operatorname{tr} I I=0$. This constraint forces $\operatorname{dim}|I I|<2$, and reasoning as in $\S 1$, $\operatorname{dim}|\mathrm{II}|=-1$ implies that $M^{2}=S^{2}=S^{n} \cap \mathbf{R}^{3} ; \operatorname{dim}|\mathrm{II}|=0$ implies that $M^{2} \subset S^{3}$. If $\operatorname{dim}|\mathrm{II}|=1$, we may take II $=\left|\left(\omega^{1}\right)^{2}-\left(\omega^{2}\right)^{2}, \omega^{1} \omega^{2}\right|$ and this system is clearly free of base points.

3. Global Theory: Chern class formulas. Given a surface $M \subset \mathbf{P}^{5}$, we would now like to calculate its inflection locus, i.e., the subvariety along which the osculating space $\tilde{\mathscr{T}}_{z}^{(2)} M$ drops rank. The key is to compute the Chern classes of various bundles which arise naturally from the geometry we've been discussing. To begin, we introduce some systematic notation and basic exact sequences.

Let $M$ be an abstract $k$-dimensional (complex) manifold. Let $T M$ and $T^{(2)} M$ be the abstract tangent bundle and second-order tangent bundle respectively (cf. [16]). In local coordinates $z^{1}, \ldots, z^{k}$, one should view sections of $T M$ as first-order differential operators $\sum b^{i}(z)\left(\partial / \partial z^{i}\right)$ and those of $T^{(2)} M$ as second-order differential operators

$$
\sum a^{i j} \frac{\partial^{2}}{\partial z^{i} \partial z^{j}}+\sum b^{i} \frac{\partial}{\partial z^{i}} .
$$

Then there is an obvious exact sequence (in general, non-split)

$$
0 \rightarrow T M \rightarrow T^{(2)} M \rightarrow \operatorname{Sym}^{2}(T M) \rightarrow 0 .
$$


Now if $M \subset \mathbf{C}^{n}$, there is a natural geometrization map

$$
\begin{gathered}
T M \stackrel{g}{\rightarrow} \mathscr{T} M \\
T^{(2)} M \stackrel{g}{\rightarrow} \mathscr{T}^{(2)} M
\end{gathered}
$$

obtained by applying the formal differential operators to the position vector on $M$; since $M$ is embedded, $\mathscr{T} M$ is naturally isomorphic with $T M$. However, $\mathscr{T}^{(2)} M$, whose fiber $\mathscr{T}_{z}^{(2)} M$ at $z \in M$ is the geometric second-order tangent space (cf. $§ 1$ ), is in general not a vector bundle.

Similarly, if $M \subset \mathbf{P}^{n}$, we let $\tilde{M} \subset \mathbf{C}^{n+1}-\{0\}$ be the cone $\pi^{-1}(M)$ and note that there is a canonical vector field $\partial / \partial t$ on $\tilde{M}$ (the so called Euler vector field, $\partial / \partial t=\sum x^{i}\left(\partial / \partial x^{i}\right)$, where $x^{0}, \ldots, x^{n}$ are the standard coordinates on $\left.\mathbf{C}^{n+1}-\{0\}\right)$. Since the tangent plane to $\tilde{M}$ is constant along rulings, we define the vector bundle $\tilde{T} M$ on $M$ by associating to $z \in M$ the tangent space to $\tilde{M}$ at any point of $\pi^{-1}(z)$. Note that then $\mathbf{P}(\tilde{T} M)$ is the bundle of tangent $\mathbf{P}^{k}$, s to $M$ in $\mathbf{P}^{n}$, and so we call $\tilde{T} M$ the abstract projective tangent bundle of $M$. The Euler sequence relates $\tilde{T} M$ to the abstract tangent bundle of $M$ (cf. [19]):

$$
0 \rightarrow \mathcal{O}_{M}(-1) \rightarrow \tilde{T} M \rightarrow T M \otimes \mathcal{O}_{M}(-1) \rightarrow 0 .
$$

Again interpreting sections of $\tilde{T} M$ as operators, observe that if $U \subset M$ is open and $f: U \rightarrow \mathbf{C}$ is holomorphic then $(\partial / \partial t)(f \circ \pi)=0$, accounting for the kernel above.

Note that if $f: U \rightarrow \mathbf{C}^{n+1}$ is a local holomorphic lifting of $M$, with local coordinates $z^{1}, \ldots, z^{k}$ on $U$, then $z^{1}, \ldots, z^{k}, t$ give local coordinates on $\pi^{-1}(U)$ and since $(\partial / \partial t)(f \circ \pi)=0$, it follows that

$$
\frac{\partial^{2}}{\partial t^{2}}(f \circ \pi)=\frac{\partial^{2}}{\partial z^{i} \partial t}(f \circ \pi)=0 .
$$

Since $\partial / \partial t$ is intrinsically defined on $\tilde{M}$, the submodule $\partial / \partial t \otimes T \tilde{M} \subset$ $T^{(2)} \tilde{M}$ is intrinsically defined as well, and we define $\tilde{T}^{(2)} M$ to be the vector bundle on $M$ with fiber

$$
\tilde{T}_{z}^{(2)} M=\left(T^{(2)} \tilde{M} /\left(\frac{\partial}{\partial t} \otimes T \tilde{M}\right)\right)_{\tilde{z}}
$$

for any $\tilde{z} \in \pi^{-1}(z)$.

This is the abstract projective second-order tangent bundle of $M$. As before, we again have the geometrization maps

$$
\begin{gathered}
\tilde{T} M \stackrel{\tilde{g}}{\rightarrow} \tilde{\mathscr{T}} M \\
\tilde{T}^{(2)} M \stackrel{\tilde{g}}{\rightarrow} \tilde{\mathscr{T}}^{(2)} M=\bigcup_{z \in M} \tilde{\mathscr{T}}_{z}^{(2)} M \subset M \times \mathbf{C}^{6}
\end{gathered}
$$


$\tilde{g}: \tilde{T} M \rightarrow \tilde{\mathscr{T}} M$ is an isomorphism, once again, since $M$ is embedded; however, $\tilde{g}: \tilde{T}^{(2)} M \rightarrow \tilde{\mathscr{T}}^{(2)} M$ is a surjection but has kernel at any inflection point.

Analogous to the classical Euler sequence (3.3), we also have a second-order version:

$$
0 \rightarrow \mathcal{O}_{M}(-1) \rightarrow \tilde{T}^{(2)} M \rightarrow T^{(2)} M \otimes \mathcal{O}_{M}(-1) \rightarrow 0 .
$$

Let us now return to the study of surfaces in $\mathbf{P}^{5}$. Suppose $M^{2} \subset \mathbf{P}^{5}$ has the property that $\operatorname{dim} \mathbf{P} \tilde{\mathscr{T}}_{z}^{(2)} M=5$ at a generic point $z \in M$; we would like to determine the locus of points at which $M$ is inflected, i.e., the set $Z=\left\{z \in M: \operatorname{dim}: \mathbf{P} \tilde{\mathscr{T}}_{z}^{(2)} M \leq 4\right\}$. If we could reembed $M \stackrel{\cong}{\rightarrow} M^{\prime}$ $\subset \mathbf{P}^{N}, N \geq 6$, so that $M^{\prime}$ is totally uninflected and so that $M=p\left(M^{\prime}\right)$ for some (linear) projection $p: \mathbf{P}^{N} \rightarrow \mathbf{P}^{5}$, then we could reason as follows. Clearly $z=p\left(z^{\prime}\right) \in M$ will be an inflection point if and only if $\mathbf{P} \tilde{\mathscr{T}}_{z^{\prime}}^{(2)} M^{\prime}$ meets the center of the projection $p$, which is a suitably chosen $\mathbf{P}_{0}^{N-6}$. Consider the second-order Gauss map $\gamma^{(2)}: M^{\prime} \rightarrow \mathbf{G}(5, N), \gamma^{(2)}(z)=$ $\tilde{\mathscr{T}}_{z}^{(2)} M^{\prime}$. The Schubert cycle $\sigma_{1} \subset \mathbf{G}(5, N)$ consists precisely of those $\mathbf{P}^{5}$ 's meeting a fixed $\mathbf{P}_{0}^{N-6} ; \sigma_{1}$ is Poincaré dual to $-c_{1}(E)$, where $E$ is the tautological bundle on $\mathbf{G}(5, N)$. We therefore deduce that $-c_{1}\left(\tilde{T}^{(2)} M\right)$ represents the divisor of inflection points of $M$, since $\tilde{T}^{(2)} M^{\prime}=p^{*} \tilde{T}^{(2)} M$. The flaw in this argument is, of course, that if $M$ is projectively normal, then it can be the image of no projection whatsoever; for example, if $M$ is a complete intersection, no such re-embedding exists. Interestingly enough, this does lead to the right formula; we now prove a beautiful result from singularity theory which gives us this formula directly.

Let $M$ be a compact, smooth manifold. We work in the formal group $K(M)$ of vector bundles on $M$; in particular, if $0 \rightarrow E^{\prime} \rightarrow E \rightarrow E^{\prime \prime} \rightarrow 0$ is an exact sequence of vector bundles, we set $E=E^{\prime}+E^{\prime \prime}$, and the total Chern class gives a homomorphism $c\left(E^{\prime}+E^{\prime \prime}\right)=c\left(E^{\prime}\right) c\left(E^{\prime \prime}\right)$. We also write $E^{\prime \prime}=E-E^{\prime}$ and $c\left(E^{\prime \prime}\right)=c(E) / c\left(E^{\prime}\right)$.

(3.5) LEMMA. Let $F$ be a vector bundle of rank $l$, and $L$ a line bundle. Then $c_{l}(L \otimes F)=c_{l}\left(F-L^{*}\right)$.

Proof. This is a formal calculation. Write $c(F)=\prod_{i=1}^{l}\left(1+\gamma_{l}\right)$ and $c(L)=1+\beta$. Then $c(L \otimes F)=\Pi\left(1+\gamma_{i}+\beta\right)$, whence

$$
c_{l}(L \otimes F)=\prod\left(\gamma_{l}+\beta\right)=\sum_{i=0}^{l} s_{l}\left(\gamma_{1}, \ldots, \gamma_{l}\right) \beta^{l-i}
$$


(where $s_{i}$ are the elementary symmetric functions). On the other hand,

$$
\begin{aligned}
c\left(F-L^{*}\right) & =\frac{c(F)}{c\left(L^{*}\right)}=\frac{1}{1-\beta} \prod\left(1+\gamma_{i}\right) \\
& =\left(1+\beta+\beta^{2}+\cdots\right) \prod\left(1+\gamma_{i}\right),
\end{aligned}
$$

so

$$
c_{l}\left(F-L^{*}\right)=\sum_{i=0}^{l} s_{i}\left(\gamma_{1}, \ldots, \gamma_{l}\right) \beta^{l-i}
$$

as required.

Let $M$ be a compact, smooth manifold, and $E, F$ vector bundles of ranks $k$ and $l$ respectively, $k \leq l$. Let $\varphi: E \rightarrow F$ be a bundle map, and let $Z=\left\{x \in M: \varphi_{x}: E_{x} \rightarrow F_{x}\right.$ is not injective $\}$ be the singular locus of $\varphi$. Let $\pi: \mathbf{P}(E) \rightarrow M$ and consider the tautological exact sequence of vector bundles on $\mathbf{P}(E)$ :

$$
0 \rightarrow L \rightarrow \pi^{*} E \rightarrow Q \rightarrow 0 .
$$

Then $\varphi$ induces a bundle map $\tilde{\varphi}: L \rightarrow \pi^{*} F$ which is zero precisely at those points $\tilde{Z} \subset \mathbf{P}(E)$ lying over $Z \subset M$.

(3.6) Theorem. (Thom-Porteous, [17].) Let $M, \varphi: E \rightarrow F$ be as above, and suppose $\tilde{\varphi}$ is transverse to the zero section of $\operatorname{Hom}\left(L, \pi^{*} F\right)$. Then $Z$ is Poincaré dual to $c_{l-k+1}(F-E)$.

Proof. With our transversality assumption it is standard (cf. [9]) that $\tilde{Z}$ is dual to $c_{l}\left(L^{*} \otimes \pi^{*} F\right)$, and so $Z$ is dual in $M$ to $\pi_{*}\left(c_{l}\left(L^{*} \otimes \pi^{*} F\right)\right)$. Since the class of the fiber of $\pi$ is $c_{k-1}(Q)$, we compute, using (3.5):

$$
\begin{aligned}
\pi_{*}\left(c_{l}\left(L^{*} \otimes \pi^{*} F\right)\right) & =\pi_{*}\left(c_{l}\left(\pi^{*} F-L\right)\right)=\pi_{*}\left(c_{l}\left(\pi^{*}(F-E)+Q\right)\right) \\
& =\pi_{*}\left(\sum_{i=0}^{l} c_{l-i}\left(\pi^{*}(F-E)\right) \cup c_{i}(Q)\right) \\
& =c_{l-k+1}(F-E) .
\end{aligned}
$$

REMARK. The transversality condition in (3.6) can be weakened considerably. So long as $Z$ has the correct dimension, the result is still correct if one includes appropriate multiplicities (which is particularly straightforward in the algebraic category). This is in essence the setting of the Poincaré-Hopf Theorem.

In order to apply this result, we will need to know the Chern classes of $\tilde{T} M$ and $\tilde{T}^{(2)} M$, and so we compute these next. 
(3.7) Proposition. Let $M \subset \mathbf{P}^{n}$ be a smooth surface, let $c_{1}, c_{2}$ denote the Chern classes of its tangent bundle, and let $H$ denote the hyperplane class. Then

$$
\begin{array}{ll}
c_{1}\left(\operatorname{Sym}^{2} T M\right)=3 c_{1}, & c_{2}\left(\operatorname{Sym}^{2} T M\right)=2 c_{1}^{2}+4 c_{1} \\
c_{1}(\tilde{T} M)=c_{1}-3 H, & c_{2}(\tilde{T} M)=c_{2}-2 c_{1} \cdot H+3 H^{2} \\
c_{1}\left(\tilde{T}^{(2)} M\right)=4 c_{1}-6 H & c_{2}\left(\tilde{T}^{(2)} M\right)=5\left(c_{1}^{2}+c_{2}\right)-20 c_{1} \cdot H+15 H^{2} .
\end{array}
$$

Proof. These are derived in a straightforward fashion using the exact sequences (3.1), (3.3), and (3.4) above.

Using this information, we can now obtain the characterization of the Veronese surface in $\mathbf{P}^{5}$ to which we referred in the introduction.

(3.8) Proposition. Let $M \subset \mathbf{P}^{5}$ be a smooth surface with the property that $\operatorname{dim} \mathbf{P} \tilde{\mathscr{T}}_{z}^{(2)} M=5$ at all $z \in M$. Then $M$ is the Veronese surface, i.e., the embedding of $\mathbf{P}^{2}$ in $\mathbf{P}^{5}$ under the complete linear system of conics.

Proof. If $\operatorname{dim} \mathbf{P} \tilde{\mathscr{T}}_{z}^{(2)} M=5$ for all $z \in M$, then we clearly have $\tilde{T}^{(2)} M$ $\cong \tilde{\mathscr{T}}^{(2)} M \cong \mathbf{C}^{6}$. Therefore $c_{1}\left(\tilde{T}^{(2)} M\right)=c_{2}\left(\tilde{T}^{(2)} M\right)=0$, so by (3.7), we have (using $K=-c_{1}$ )

$$
\begin{gathered}
4 K=-6 H \\
c_{1}^{2}+c_{2}=3 H^{2}=3 d,
\end{gathered}
$$

where $d=$ degree $M$. By the Riemann-Roch theorem for surfaces, $\chi\left(\mathcal{O}_{M}\right)$ $=1-q+p_{g}=3 d / 12=d / 4$. Since, by (3.9), the canonical bundle is negative, $p_{g}=h^{2}\left(\mathcal{O}_{M}\right)=h^{0}(\mathcal{O}(K))=0$, and so $\chi\left(\mathcal{O}_{M}\right)=1-q \leq 1$, whence $d \leq 4$. Since $M$ lies in no hyperplane, we must have $d=4$, and $K^{2}=9, c_{2}=3$. The hyperplane sections of $M$ are quartic curves in $\mathbf{P}^{4}$, hence rational, and so $M$ itself must be rational; since the (topological) Euler characteristic is 3, $M$ must be $\mathbf{P}^{2}$, embedded as the Veronese. (For more details, cf. [9, p. 525].) Indeed, the only other smooth surface of degree 4 in $\mathbf{P}^{5}$ is a rational ruled surface which we shall encounter shortly.

REMARK. This can be interpreted as the first generalization of the classical Plücker-type result that the only curve in $\mathbf{P}^{n}$ which is totally uninflected is the rational normal curve; cf. [8] for higher-dimensional results. 
We now derive a simple formula which will be the key to our remaining work. Suppose $M \subset \mathbf{P}^{5}$ is a hypo-osculating surface, i.e., $\operatorname{dim} \mathbf{P} \tilde{\mathscr{T}}_{z}^{(2)} M \leq 4$ for all $z \in M$. If $\operatorname{dim} \mathbf{P} \tilde{\mathscr{T}}_{z}^{(2)} M \leq 3$ it follows that $M$ is a plane or else developable, and there exists no smooth developable variety (cf. [10]). Therefore, we assume this dimension is generically 4 , and would like an expression involving Chern classes for the locus of inflection points, i.e., $\left\{z \in M\right.$ : $\left.\operatorname{dim} \mathbf{P} \tilde{\mathscr{T}}_{z}^{(2)} M \leq 3\right\}$.

Since $M$ is hypo-osculating, we know that the geometrization map

$$
\tilde{g}: \tilde{T}^{(2)} M \rightarrow M \times \mathbf{C}^{6}
$$

generically has one-dimensional kernel; let us assume moreover that this extends to give a one-dimensional smooth subbundle $\mathscr{L}$ of $\tilde{T}^{(2)} M$. (This is a nontrivial condition, as the example

$$
x \frac{\partial^{2}}{\partial x^{2}}+y \frac{\partial^{2}}{\partial y^{2}} \quad \text { at }(x, y)=(0,0)
$$

shows.) To apply (3.6) we observe that the map

$$
\varphi: Q=\tilde{T}^{(2)} M / \mathscr{L} \rightarrow \mathbf{C}^{6}
$$

induced by $\tilde{g}$ is generically an injection, and so its singularities are represented by $c_{2}\left(\mathbf{C}^{6}-Q\right)=\left(c_{1}^{2}-c_{2}\right)(Q)$.

In this event we find the following count:

(3.10) Proposition. Suppose $M \subset \mathbf{P}^{5}$ is degree $d$ and hypo-osculating with $\operatorname{dim} \mathbf{P} \tilde{\mathscr{T}}_{z}^{(2)} M=4$ generically, and suppose the kernel $\mathscr{L}$ of the geometrization map globalizes smoothly. Then the degree of the inflection locus is given by

$$
\iota=11 c_{1}^{2}-5 c_{2}+21 d-28 c_{1} \cdot H+c_{1}(\mathscr{L}) \cdot\left(6 H-4 c_{1}\right) .
$$

Proof.

$$
\begin{aligned}
c_{2}\left(\mathbf{C}^{6}-Q\right) & =\left(c_{1}^{2}-c_{2}\right)(Q)=\left(c_{1}^{2}-c_{2}\right)\left(\tilde{T}^{(2)} M / \mathscr{L}\right) \\
& =\left(c_{1}^{2}-c_{2}\right)\left(\tilde{T}^{(2)} M\right)-c_{1}\left(\tilde{T}^{(2)} M\right) \cdot c_{1}(\mathscr{L}) .
\end{aligned}
$$

Now we substitute the formulas from (3.7) to obtain

$$
\begin{aligned}
\iota= & \left(4 c_{1}-6 H\right)^{2}-\left(5\left(c_{1}^{2}+c_{2}\right)-20 c_{1} \cdot H+15 d\right) \\
& -c_{1}(\mathscr{L}) \cdot\left(4 c_{1}-6 H\right) \\
= & 11 c_{1}^{2}-5 c_{2}+21 d-28 c_{1} \cdot H+c_{1}(\mathscr{L}) \cdot\left(6 H-4 c_{1}\right) .
\end{aligned}
$$


REMARK. This result may be viewed as a generalization of work of Pohl [16, §IV].

In the sequel the following remark will prove useful.

(3.11) Since $M \subset \mathbf{P}^{5}$ is embedded, the line bundle $\mathscr{L}$ projects, by means of the sequences (3.1) and (3.4), to an isomorphic bundle $\overline{\mathscr{L}} \subset$ $\operatorname{Sym}^{2}(T M) \otimes \mathcal{O}_{M}(-1)$.

This map $\mathscr{L} \rightarrow \overline{\mathscr{L}}$ is essentially a twisted symbol map. In particular, so far as Chern class calculations are concerned, of course, we have $c_{1}(\mathscr{L})=c_{1}(\overline{\mathscr{L}})$.

The crucial point for much of our remaining work is the observation that if $\varphi: E \rightarrow F$ is a vector bundle map of constant rank, then $\operatorname{ker} \varphi \subset E$ is a subbundle; this is a straightforward consequence, for example, of the rank theorem (either smooth or holomorphic). In the event that $M \subset \mathbf{P}^{5}$ is perfectly hypo-osculating, the geometrization map $\tilde{g}$ has rank five everywhere, and so $\mathscr{L}=\operatorname{ker} g$ is automatically globally defined. In this case, our formula (3.10) must yield $\iota=0$, since there can be no inflection point. If the numerical characters appearing in (3.10) are such that $\iota=0$ is arithmetically impossible, then it follows that no such perfectly hypoosculating embedding of $M \subset \mathbf{P}^{5}$ exists. Of course, to perform this computation one needs to get one's hands on $\mathscr{L}$ in some intrinsic manner, and this is easily done only in the case of ruled surfaces, to which we turn next.

4. Inflection points of ruled surfaces. From our discussion of the local theory in $\$ 2$, it is clear that a ruled surface $M$ is hypo-osculating; on the other hand, we should expect, according to our results of $\S 3$, that for most ruled surfaces the dimension of the osculating space will drop at a finite number of points. Our aim in this section is to compute the number of inflection points and classify the ruled surfaces which are uninflected, using the formula at the end of the preceding section.

We being with the case of rational ruled surfaces, recalling here the salient facts (cf. [9]). First of all, any such surface is abstractly the blow-up of one of the relatively minimal models $S_{n}=\mathbf{P}(\mathcal{O} \oplus \mathcal{O}(n)) \stackrel{p}{\rightarrow} \mathbf{P}^{1}, n \geq 0$. Since we know $M$ is geometrically ruled, there can be no exceptional curves. Next, $\operatorname{Pic}\left(S_{n}\right) \cong \mathbf{Z} \oplus \mathbf{Z}$, with generators the base $C_{0}$ and the fiber $F$; the intersection pairing is given by $C_{0} \cdot C_{0}=n, C_{0} \cdot F=1, F \cdot F=0$. In order to embed $S_{n}$ in $\mathbf{P}^{N}$ as a geometrically ruled surface by the linear system $\left|k C_{0}+l F\right|$, we see that we must have $k=1$. Then it is easy to 
check, using the Riemann-Roch Theorem, that under the complete linear system $\left|C_{0}+l F\right|, S_{n}$ embeds in $\mathbf{P}^{N}, n=n+2 l+1$, as a surface of degree $\left(C_{0}+l F\right)^{2}=n+2 l$; by definition, $H=C_{0}+l F$. A straightforward application of the adjunction formula yields the formula $K=-2 C_{0}$ $+(n-2) F$ for the canonical bundle of $S_{n}$.

In order to evaluate the formula (3.10) for the number of inflection points, it remains for us to determine the line bundle $\mathscr{L}$. Let $E \subset T M$ be the subbundle whose fiber at $z$ is the tangent space to the ruling through $z$. Since this tangent space is constant along the ruling, we see that $E^{2} \subset \operatorname{Sym}^{2}(T M)$ is the twist by $\mathcal{O}_{M}(1)$ of the bundle $\overline{\mathscr{L}}$ appearing in (3.11). Therefore, $\overline{\mathscr{L}} \cong E^{2} \otimes \mathcal{O}_{M}(-1)$, whence $\mathscr{L} \cong E^{2} \otimes \mathcal{O}_{M}(-1)$, and so $c_{1}(\mathscr{L})=2 c_{1}(E)-H$. Recalling that $M$ is a $\mathbf{P}^{1}$-bundle over $\mathbf{P}^{1}$, we have the sequence

$$
0 \rightarrow E \rightarrow T M \rightarrow p^{*} T \mathbf{P}^{1} \rightarrow 0,
$$

and so it follows that $c_{1}(E)=c_{1}-2 F$. Therefore

$$
\begin{aligned}
c_{1}(\mathscr{L}) & =2\left(c_{1}-2 F\right)-\left(C_{0}+l F\right)=-2 K-(4+l) F-C_{0} \\
& =-2\left(-2 C_{0}+(n-2) F\right)-(4+l) F-C_{0} \\
& =3 C_{0}-(2 n+l) F .
\end{aligned}
$$

Plugging this into the magical formula (3.10), along with $c_{2}=4$ and the expressions for $K, H, d$, we obtain the number $\iota=3(n+2 l-4)$. Now, we pointed out earlier that the complete linear system $\left|C_{0}+l F\right|$ embeds $M$ in $\mathbf{P}^{n+2 l+1}$. When we restrict to a five-dimensional sub-linear system (or project $\mathbf{P}^{n+2 l+1}$ to $\mathbf{P}^{5}$ ), nothing in the above computation is changed. We therefore have the following

(4.1) Proposition. Let $S_{n} \subset \mathbf{P}^{5}$ be embedded as a ruled surface of degree $n+2 l$. Then if the inflection locus is zero-dimensional, it has degree $\iota=3(n+2 l-4)=3(d-4)$.

It is now easy to characterize the uninflected surfaces. In order to have $d=n+2 l=4$, there are three possiblities:

(a) $n=4, l=0$. The corresponding map fails to be an embedding, as the infinity section (i.e., the unique curve on $S_{4}$ of self-intersection -4) collapses to a point. (To see this, note that in order to have self-intersection $-n$ on the surface $S_{n}, C_{\infty}$ must be linearly equivalent to $C_{0}-n F$. In this case, we have $C_{\infty}=C_{0}-4 F$, and $C_{\infty} \cdot H=\left(C_{0}-4 F\right) \cdot C_{0}=4-4$ $=0$.)

(b) $n=2, l=1$. This surface is unacceptable for more subtle reasons. Note that in this case, $C_{\infty}=C_{0}-2 F$ and $C_{\infty} \cdot H=1$, so that $C_{\infty}$ maps 
to a line. But along this line, $\mathbf{P} \tilde{T}^{(2)} M$ can be only three-dimensional, and so this surface has (at least) a line of inflection points.

(c) $n=0, l=2$. Here we embed $S_{0}=\mathbf{P}^{1} \times \mathbf{P}^{1}$ by the linear system $|\mathcal{O}(1) \otimes \mathcal{O}(2)| ;$ in coordinates, we have

$$
\begin{gathered}
\mathbf{P}^{1} \times \mathbf{P}^{1} \rightarrow \mathbf{P}^{5} \\
(s, t) \rightarrow\left(s, t, s^{2}, s t, s^{2} t\right) .
\end{gathered}
$$

Thus far, the surface (4.2) is the only uninflected ruled surface.

We now turn to the case of irrational ruled surfaces, i.e., $\mathbf{P}^{1}$-bundles over curves $C$ of arbitrary genus $g \geq 1$. One's intuition is that with increasing algebro-geometric complexity must come greater curvature and hence inflectionary behavior. Indeed, it is not hard to give an explicit formula for the number of inflection points.

(4.3) Proposition. Let $M \subset \mathbf{P}^{5}$ be as ruled surface of genus $g$, i.e., $M$ is a $\mathbf{P}^{1}$-bundle over a curve $C$ of genus $g$. Assuming it has only finitely many inflection points, then it has precisely $\iota=3(d+4(g-1))$ such.

From this, of course, follows our main result of this section:

(4.3) THEOREM. There is a unique (smooth) ruled surface in $\mathbf{P}^{5}$ which is uninflected, i.e., the surface (4.2) above.

Proof of Proposition. It is not hard to see (e.g., from a spectral sequence argument) that $H_{2}(M) \cong \mathbf{Z} \oplus \mathbf{Z}$, and, as in the rational case, the group is generated by a copy $C_{0}$ of the base and the fiber $F$. Letting $C_{0}^{2}=n$, which is necessarily positive by the Hodge index theorem, we find that $K=-2 C_{0}+(2 g-2+n) F$; this follows from the adjunction formula, as in the rational case. Then $K^{2}=c_{1}^{2}=8(1-g)$. Letting $H=C_{0}$ $+l F$, we compute $K \cdot H=2 g-2 l-n-2$ and $d=H^{2}=n+2 l$. The remaining piece is

$$
c_{1}(\mathscr{L})=2\left(c_{1}-(2-2 g) F\right)-H=3 C_{0}-(2 n+l) F,
$$

so that

$$
c_{1}(\mathscr{L}) \cdot(6 H+4 K)=10 n+20 l+24(g-1) .
$$

Putting this all together, we find

$$
\begin{aligned}
\iota= & 11 c_{1}^{2}-5 c_{2}+21 d+28 K \cdot H+c_{1}(\mathscr{L}) \cdot(6 H+4 K) \\
= & 11 \cdot 8(1-g)-5 \cdot 4(1-g)+21(n+2 l) \\
& +28(2 g-2 l-n-2)+(10 n+20 l+24(g-1)) \\
= & 3(n+2 l+4(g-1))=3(d+4(g-1)) .
\end{aligned}
$$


5. Inflection points of surfaces of heat and wave equation type. While the geometry of ruled surfaces is quite explicit, we have far less control a priori over surfaces satisfying the heat or wave equations. We begin with two simple, but illuminating, examples.

EXAMPLE 1 (wave equation type). Consider the rational surface $M \subset$ $\mathbf{P}^{5}$ given by the rational map

$$
\begin{aligned}
& \varphi: \mathbf{P}^{2}--\rightarrow \mathbf{P}^{5} \\
&(1, u, v) \rightarrow\left(u, v, u^{2}, v^{2}, u^{2} v, u v^{2}\right) .
\end{aligned}
$$

Togliatti [22] shows that this surface is generically of wave equation type; it is a smooth surface of degree six whose generic hyperplane section is a curve of genus one. Indeed, one obtains the linear system by considering cubics in $\mathbf{P}^{2}$ with three assigned base points (this gives a linear system of dimension six) and then requiring that every other point of $\mathbf{P}^{2}$ be a triple point of some cubic (cf. the beginning of the introduction). It is easy to check then that $M$ is biholomorphic to the blow-up of $\mathbf{P}^{2}$ at three points; each of the exceptional curves and each of the coordinate axes maps under $\varphi$ to a line in $M \subset \mathbf{P}^{5}$. Indeed, one can check that there are six points of intersection of these assorted lines, and at each of these six points, $\operatorname{dim} \mathbf{P} \tilde{\mathscr{T}}^{(2)} M \leq 3$. It follows that $M$ is hypo-osculating, but exhibits further inflectionary behavior.

EXAMPLE 2 (heat equation type). The simplest example here is the image $M$ of the rational map

$$
\begin{gathered}
f: \mathbf{P}^{2} \rightarrow \mathbf{P}^{5} \\
(x, y, 1) \rightarrow\left(1,2 x+y^{2}, y, y^{3}+6 x y, y^{4}+12 x y^{2}+12 x^{2}\right. \\
\left.y^{5}+20 x y^{3}+60 x^{2} y\right) .
\end{gathered}
$$

Note that $\partial^{2} f / \partial y^{2}=\partial f / \partial x$. We claim, however, that $M$ is singular. In homogeneous coordinates, $f$ is given by the following linear subsystem of $\left|\mathcal{O}_{\mathbf{P}^{2}}(5)\right|$ :

$$
\begin{aligned}
& L=\mid z^{5}, 2 x z^{4}+y^{2} z^{3}, y z^{4}, y^{3} z^{2}+6 x y z^{3}, y^{4} z+ 12 x y^{2} z^{2}+12 x^{2} z^{3}, \\
& y^{5}+20 x y^{3} z+60 x^{2} y z^{2} \mid,
\end{aligned}
$$

from which it is clear that

(i) $p=(1,0,0)$ is a base point of $L$, and

(ii) $C=\{z=0\}$ blows down to $(0, \ldots, 0,1)$.

We therefore begin by blowing up $p$ twice to define the mapping at $(1,0,0)$. 

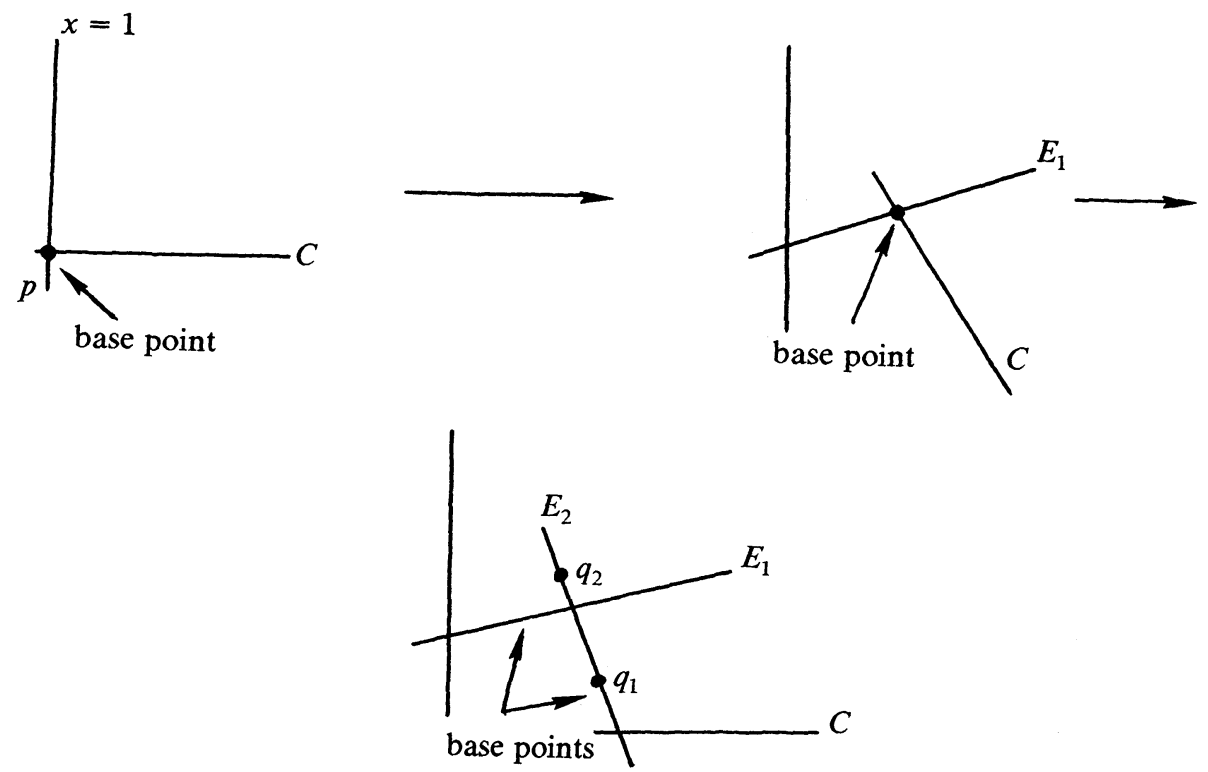

At this juncture there are two remaining base points $q_{1}, q_{2}$ on the exceptional curve $E_{2}$. Upon blowing them up, we have the picture

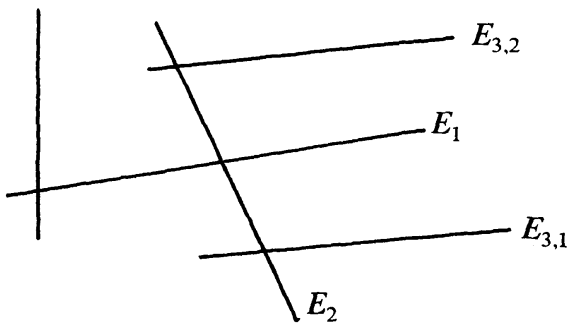

It is a straightforward computation to check at this stage that $C$ and $E_{2}$ blow down to $(0, \ldots, 0,1)$ and, more important, that $E_{1}, E_{3,1}$ and $E_{3,2}$ all map to the same line $\ell$ in $M$. From this our claim follows immediately. For suppose the surface $M$ were smooth, or, indeed, just normal; then by Zariski's Main Theorem (cf. [11, p. 280]), the fibers of the birational map $\tilde{\mathbf{P}}^{2} \rightarrow M \subset \mathbf{P}^{5}$ would be connected. But the preimage of any point of $\ell$ other than $(0, \ldots, 0,1)$ is disconnected. Therefore $M$ is singular.

With these examples in mind, we are led to generalize (4.3):

(5.1) Conjecture. The unique perfectly hypo-osculating surface in $\mathbf{P}^{5}$ is the rational ruled surface (4.2). 
As evidence for this conjecture, we attempt to compute explicitly the number of inflection points (assuming it is finite) of a hypo-osculating surface in $\mathbf{P}^{5}$. Before doing so, we make some preliminary observations.

(5.2) LEMMA. If the surface $M \subset \mathbf{P}^{5}$ is of heat equation type, there is a distinguished line subbundle of TM. If $M$ is of wave equation type, then the tangent bundle splits (analytically), passing, if necessary, to a double cover $\tilde{M}$ of $M$.

Proof. In the former case, we are interested in changes of local coordinates $(x, y) \rightarrow\left(x^{\prime}, y^{\prime}\right)$ under which the operator $\partial^{2} / \partial y^{2}-\varphi(\partial / \partial x)$ transforms to $\partial^{2} / \partial y^{\prime 2}-\varphi^{\prime}\left(\partial / \partial x^{\prime}\right)$. It is an elementary calculation using the chain rule that we must have $\partial / \partial y^{\prime}=a(x, y)(\partial / \partial y)$, and hence the line bundle spanned by $\partial / \partial y$ is intrinsically determined. Similarly, in the latter case, in order for $\partial^{2} / \partial x \partial y$ to transform to $\partial^{2} / \partial x^{\prime} \partial y^{\prime}$, both $\partial / \partial x$ and $\partial / \partial y$ must be distinguished, and so we obtain a local direct sum decomposition of $T M$; passing, if necessary, to a double cover $\pi: \tilde{M} \rightarrow M$, $\pi^{*}(T M)$ splits globally.

REMARK. The existence of the trace mapping $\pi_{*}: H^{*}(\tilde{M}, Q) \rightarrow$ $H^{*}(M, Q)$ shows that $\pi^{*}: H^{*}(M, Q) \rightarrow H^{*}(\tilde{M}, Q)$ is injective. Hence, for the purpose of Chern classes calculations on $M$, we infer from a splitting $\pi^{*}(T M) \cong \pi^{*}\left(L_{1}\right) \oplus \pi^{*}\left(L_{2}\right)$ that $c_{1}(T M)=c_{1}\left(L_{1}\right)+c_{1}\left(L_{2}\right)$.

We saw in example 1 that a surface of wave equation type could contain lines. In fact, one expects such a degeneration of the second fundamental form to occur-along a divisor the linear system II acquires base points. This can be interpreted bundle-theoretically as follows. Where the wave equation is valid, we have a splitting $\pi^{*} T M=$ $\pi^{*}\left(L_{1} \oplus L_{2}\right)$; now when $\partial^{2} / \partial x \partial y$ degenerates along an effective divisor $D$ to the operator $\partial^{2} / \partial y^{2}-\varphi(\partial / \partial x)$, there is still a globally defined subbundle $L_{2} \subset T M$ correspnoding to $\partial / \partial y$. Off the divisor $D$, the other distinguished subbundle $L_{1}$ is a complement, but along $D$ we have $L_{1}=L_{2}$. Indeed, following this reasoning, we infer that

(5.3) the divisor $D$ represents the class $c_{1}\left(L_{1}^{*} \otimes\left(T M / L_{2}\right)\right)$.

We are now in a position to deduce the following

(5.4) Proposition. There is no smooth perfectly hypo-osculating surface generically of wave equation type which contains lines. 
Proof. Suppose the divisor $D$ above consists of disjoint lines (they are necessarily disjoint, for whenever two lines meet, the osculating space drops dimension further). It is clear from our analysis of the ruled surface case that along a line $\ell \subset M,\left.T \ell \cong L_{2}\right|_{\ell}$, and therefore, $\left.T D \cong L_{2}\right|_{D}$. Now, the adjunction formula gives

$$
\left.\left.N_{D} \cong[D]\right|_{D} \cong K_{M}^{*}\right|_{D} \otimes K_{D},
$$

while from (5.3) we infer

$$
[D] \cong L_{1}^{*} \otimes\left(T M / L_{2}\right) \cong K_{M}^{*} \otimes L_{1}^{*} \otimes L_{2}^{*},
$$

and so restricting to $D,\left.\left.[D]\right|_{D} \cong\left(K_{M}^{*} \otimes\left(L_{2}^{*}\right)^{2}\right)\right|_{D}$. Therefore

$$
K_{D}=\left.T^{*} D \cong\left(L_{2}^{*}\right)^{2}\right|_{D} ;
$$

but we observed earlier that $\left.T^{*} D \cong L_{2}^{*}\right|_{D}$, and so $\left.L_{2}\right|_{D}$ must be trivial, whence $T D$ is trivial; this is an obvious contradiction.

We next give explicit Plücker-type formulas for the number of inflection points on a surface which is a globally of wave or of heat equation type.

(5.5) Theorem. Suppose $M$ is globally of wave equation type. Then if it has finitely many inflection points, it has

$$
\iota=7 K^{2}-5 c_{2}+15 d+18 K \cdot H .
$$

Suppose $M$ is globally of heat equation type. If its third fundamental form is given by (2.13), $|\mathrm{III}|=\left|\left(\omega^{1}\right)^{2} \omega^{2}\right|$, then

$$
\iota=-\left(5\left(K^{2}+c_{2}\right)+9 d+16 K \cdot H\right)
$$

if its third fundamental form is given by (2.11), $|\mathrm{III}|=\left|\left(\omega^{1}\right)^{3}\right|$, then

$$
\iota=-\frac{1}{5}\left(K^{2}+25 c_{2}-3 d+12 K \cdot H\right) \text {. }
$$

Proof. Suppose $M$ is of wave equation type. By (5.2) we have a splitting $\pi^{*} T M=\pi^{*}\left(L_{1} \oplus L_{2}\right)$ and clearly the kernel of the local geometrization map $g: T^{(2)} M \rightarrow \mathrm{C}^{5}$ is isomorphic to $L_{1} \otimes L_{2}$; it follows from (3.4) that $\mathscr{L} \cong L_{1} \otimes L_{2} \otimes \mathcal{O}_{M}(-1)$. So $c_{1}(\mathscr{L})=c_{1}-H=-(K+H)$. Substituting this in (3.10) gives (5.5a).

In the case of the heat equation, we see that under the projection $T^{(2)} M \rightarrow \operatorname{Sym}^{2}(T M)$ the heat equation operator $\left(\partial^{2} / \partial y^{2}\right)-\varphi(\partial / \partial x)$ 
maps to $\partial^{2} / \partial y^{2}$; bundle theoretically, we therefore have $\mathscr{L} \cong L_{2}^{2} \otimes$ $\mathcal{O}_{M}(-1)$, and so $c_{1}(\mathscr{L})=2 c_{1}\left(L_{2}\right)-H$. To compute $c_{1}\left(L_{2}\right)$, however, we refer to our possible standard forms for the third fundamental form. We have III: $\operatorname{Sym}^{3}(T M) \rightarrow\left(\mathbf{C}^{6} / \tilde{T}^{(2)} M\right) \otimes \mathcal{O}_{M}(1)=Q$, and considering the two cases $|\mathrm{III}|=\left|\left(\omega^{1}\right)^{2} \omega^{2}\right|,\left|\left(\omega^{1}\right)^{3}\right|$, we find that III induces isomorphisms

(i) $L_{1}^{2} \otimes L_{2} \stackrel{\cong}{\rightarrow} Q$

(ii) $L_{1}^{3} \cong Q$.

Computing Chern classes, using the relevant exact sequences, we find that in case (i) $c_{1}(\mathscr{L})=-(4 K+5 H)$ and in case (ii) $c_{1}(\mathscr{L})=$ $-\frac{1}{5}(14 K+17 H)$. Formulas $(5.5 \mathrm{~b})$ and $(5.5 \mathrm{c})$ now follow by direct calculation.

We now proceed to compute the number of inflection points on general hypo-osculating surfaces in $\mathbf{P}^{5}$. We follows Kodaira's classification scheme $([\mathbf{1}],[\mathbf{1 4}])$ :

(5.6) THEOREM (Kodaira). Let $M$ be a smooth minimal algebraic surface (i.e., $M$ contains no curve of self-intersection -1). Then $M$ is isomorphic to one of the following:

(I) a surface with $p_{g}=0$; i.e.,

(a) $\mathbf{P}^{2}$,

(b) a ruled surface,

(c) an Enriques surface, or

(d) a hyperelliptic surface;

(II) a $K$-3 surface $(K=0, q=0)$;

(III) an abelian surface;

(IV) an elliptic surface;

(V) a surface of general type.

It is now a matter of calculation to compute the number $\iota$ given by (5.5) for most of these cases:

(Ia) wave: $\iota=15 d-6$

heat: (i) $\iota=-(6+9 d)$

(ii) $\iota=-\frac{1}{5}(48-3 d)$

(Ib) Suppose that $M$ is (abstractly) a ruled surface of genus $g$, embedded in $\mathbf{P}^{5}$ by a subsystem of the linear system $\left|k C_{0}+l F\right|, k \geq 2$, $l \geq 1$. Using the notation of $\S 4$, we find the formulas:

wave: $\iota=36(g-1)(k-1)+l(30 k-36)+n k(15 k-18)$

heat: (i) $\iota=-\{20(3-2 g)+d(9-16 / k)+32 k(g-1)\}$

(ii) $\iota=-\frac{1}{5}\{83-8 \mathrm{~g}-3 \mathrm{~d}(1+4 / k)+24 k(g-1)\}$. 
(Ic) If $M$ is an Enriques surface, then $q=0$ and $2 K=0$. Therefore $K^{2}=c_{1}^{2}=0$ and the Riemann-Roch Theorem implies that $1=\chi\left(\mathcal{O}_{M}\right)$ $=\frac{1}{12}\left(c_{1}^{2}+c_{2}\right)$, whence $c_{2}=12 . K \cdot H=0$ since $K$ is of order 2 . Therefore, we have:

wave: $\iota=15(d-4)$

heat: (i) $\iota=-(60+9 d)$

(ii) $\iota=-60+\frac{3}{5} d$.

(Id) If $M$ is hyperelliptic, $q=1$ and $\chi\left(\mathcal{O}_{M}\right)=0$. Since $K$ is again of finite order (cf. [9, p. 585 ff.]), $K^{2}=c_{2}=0$; similarly, $K \cdot H=0$. Hence,

wave: $\iota=15 d$

heat: (i) $\iota=-9 d$

(ii) $\iota=\frac{3}{5} d$.

(II) Suppose $M$ is a $K-3$ surface. Then $K=0$, so $\chi\left(\mathcal{O}_{M}\right)=2$ and $c_{2}=24$. Then

$$
\begin{aligned}
& \text { wave: } \iota=15(d-8) \\
& \text { heat: }(\mathrm{i}) \iota=-(9 d+120) \\
& \text { (ii) } \iota=\frac{3}{5} \mathrm{~d}-120 .
\end{aligned}
$$

(III) If $M$ is an abelian surface, $K=0, c_{2}=0$, and so wave: $\iota=15 d$

heat: (i) $\iota=-9 d$

(ii) $\iota=\frac{3}{5} d$.

(IV) If $M$ is elliptic, i.e., there is $\pi: M \rightarrow C$ with elliptic fibers and $g$ = genus $C \geq 1$, then, first of all, the topological Euler characteristic $c_{2}=\Sigma \chi\left(F_{i}\right)$, where the $F_{i}$ are the singular fibers of $\pi$. Moreover, from the structure theory of elliptic surfaces (cf. [13], [9, p. 572]), we know that the canonical bundle is obtained by pullback from $C$. In particular, $K_{M}=\pi^{*} L$ and $\operatorname{deg} L \geq 2 g-2+\chi\left(\mathcal{O}_{M}\right) \geq c_{2} / 12$. Moreover, $K^{2}=0$ and $K \cdot H=$ $(\operatorname{deg} L) F \cdot H$, where $F$ is a generic fiber; since $F \subset \mathbf{P}^{5}$ is not rational, $F \cdot H=\operatorname{deg} F \geq 6$. It also follows from [13] that $c_{2} \geq 0$, and so we can give a bound for $\iota$.

$$
\text { wave: } \begin{aligned}
\iota & =-5 c_{2}+15 d+18(K \cdot H) \\
& \geq 15 d-5 c_{2}+18\left(c_{2} / 12\right)(6)=15 d+4 c_{2}>0 .
\end{aligned}
$$

heat: (i) $\iota=-\left(5 c_{2}+9 d+16 K \cdot H\right)<0$

(ii) $\iota=\frac{3}{5} d-5 c_{2}-\frac{12}{5} K \cdot H$.

(V) In the case of surfaces of general type, $K^{2}>0$ and so, by Miyaoka's results [15] $3 c_{2} \geq K^{2}, c_{2}>0$ as well, and $K \cdot H>0$. We see therefore that for the first case of the heat equation, $\iota<0$, but in the other two cases, the formulas (5.5) stand basically unsimplified.

We have dealt so far only with minimal surfaces. It is a fundamental fact in the theory of algebraic surfaces (cf. [9]) that blowing up a surface 
at a point increases $c_{2}$ by 1 and decreases $K^{2}$ by 1 . The effect on the number $\iota$ of inflection points is therefore easily computed. In the wave equation case, $7 K^{2}-5 c_{2}$ decreases by 12 , while $18 K \cdot H$ increases by 18 (degree of the exceptional curve), accounting for a net increase of at least 24 (cf. (5.3)). In the heat equation cases, in (i) $\iota$ decreases by 16(degree of the exceptional curve), and in (ii) $K^{2}+25 c_{2}$ decreases by 24 while $12 K \cdot H$ increases by at least 24 , whence $\iota$ decreases as well.

Ultimately we would like to classify all perfectly hypo-osculating surfaces, and so we need to list the possible cases above for $\iota=0$. Since the arithmetic sometimes allows $\iota=0$, we have a few special arguments to offer. Note that $\iota<0$ is impossible.

(Ia) $T \mathbf{P}^{2}$ has no holomorphic subbundle, so (5.2) eliminates this case.

(Ib) One checks with tedious arithmetic arguments that $\iota=0$ is impossible.

(Ic) Since surfaces of degree 4 in $\mathbf{P}^{5}$ are classified (cf. proof of (3.8)), we eliminate the wave equation case. However, $d=100$ is a possible solution in the heat equation case.

(Id) $d=200$ is a priori a possible solution for the latter case.

(II) $d=8$ for the wave equation and $d=200$ for the heat equation are $a$ priori possibilities. We eliminate the former by differential geometric means, using the following Bochner-type vanishing theorem proved by using Yau's Ricci-flat metric:

(5.7) THEOREM (Kobayashi [12]). If $M$ is a (Kähler) $K-3$ surface, then $H^{0}\left(\operatorname{Sym}^{r} T M\right)=H^{0}\left(\operatorname{Sym}^{r} T^{*} M\right)=0$ for all $r \geq 1$.

(5.8) Proposition. There is no holomorphic splitting of the tangent bundle of a $K-3$ surface.

Proof. Suppose $T M \cong L_{1} \oplus L_{2}$ (recall that a $K-3$ surface is simply connected). Since $K_{M}=0, L_{2}=L_{1}^{*}$, and so $\operatorname{Sym}^{2}(T M) \cong L_{1}^{2} \oplus\left(L_{1}^{*}\right)^{2} \oplus$ $\mathcal{O}_{M}$ which clearly has a nontrivial section, contradicting (5.7).

Therefore, no $K-3$ surface can be globally of wave equation type. Moreover, if $M$ is the blow-up of a $K-3$ surface at $l$ points and is of wave equation type, then it follows from our earlier discussion that $\iota=15 d-$ $120-12 l+18 \sum \operatorname{deg} E_{i}$, where $E_{1}, \ldots, E_{l}$ are the exceptional curves, and $\operatorname{deg} E_{\imath} \geq 2$. Clearly, when $d \geq 8, l \geq 1, \iota>0$. One checks directly that for $d=5,6,7, l \geq 1$, there is no solution to $\iota=0$.

(III) Clearly $\iota=0$ is impossible. 
(IV) The inequalities we gave earlier show that there is no uninflected elliptic surface, except perhaps of the second heat equation type.

(V) Surfaces of general type are still basically intractable. However, one has the following result of Kobayashi's based on Aubin-Yau and an analysis of holonomy groups:

(5.9) THEOREM ([12]). Let $M^{n}$ be a compact $n$-dimensional Kähler manifold with positive canonical bundle $K_{M}$ and finite fundamental group. Then $H^{0}\left(\operatorname{Sym}^{n r} T M \otimes K_{M}^{r}\right)=0$ for $r>0$, unless the universal covering space of $M$ is biholomorphic to the bidisk.

Therefore, reasoning as we did in (5.8), we have the following

(5.10) Proposition. If $M$ is a surface of general type whose universal covering space is not biholomorphic to the bidisk, then $M$ cannot be globally of wave equation type:

Proof. If $T M \cong L_{1} \oplus L_{2}$, then $K_{M} \cong L_{1}^{*} \otimes L_{2}^{*}$ and so $\mathrm{Sym}^{2} T M \otimes$ $K_{M} \supset\left(L_{1} \otimes L_{2}\right) \otimes\left(L_{1} \otimes L_{2}\right)^{*} \cong \mathcal{O}_{M}$. The same argument applies, if necessary, on passing to the double cover.

REMARKS. The Plücker-type formulas provide convincing evidence for (5.1). Aside from a small number of numerical cases which remain, the major gaps are the surfaces of general type and the degeneration arguments required to finish the proof (unfortunately, when we try to correct our formulas (5.5) by applying (5.3), the signs are uncooperative).

\section{REFERENCES}

[1] E. Bombieri and D. Husemoller, Classification and embeddings of surfaces, Proc. Symp. Pure Math., 29 (Arcata), Amer. Math. Soc., (1975), 329-420.

[2] E. Cartan, Leçons sur la Géométrie Projective Complexe, Gauthiers-Villars, 1931.

[3] __ Sur les variétés de courbure constante d'un espace euclidien ou non euclidien, Oeuvres Complètes, III, vol. 1, 321-432.

[4] __ Sur la déformation projective des surfaces, loc. cit., 441-539.

[5] S. S. Chern, On the minimal immersions of the two-sphere in a space of constant curvature, Problems in Analysis, Princeton Univ. Press, 1970, 27-40.

[6] G. Darboux, Leçons sur la théorie générale des surfaces, vol. 1, 2, Chelsea, 1972.

[7] E. Feldman, Geometry of Immersions, I, Trans. Amer. Math. Soc., 120 (1965), 185-224.

[8] W. Fulton, S. Kleiman, R. Piene and H.-S. Tai, Some intrinsic and extrinsic characterizations of projective space, preprint.

[9] P. Griffiths and J. Harris, Principles of Algebraic Geometry, John Wiley and Sons, 1978. 
[10] Algebraic Geometry and Local Differential Geometry, Ann. scient. Ec. Norm. Sup., 12 (1979), 355-452.

[11] R. Hartshorne, Algebraic Geometry, Springer-Verlag, 1977.

[12] S. Kobayashi, First Chern class and holomorphic tensor fields, J. Math. Soc. Japan, 32 (1980), 325-329.

[13] K. Kodaira, On compact analytic surfaces, II, Ann. Math., 77 (1963), 563-626.

[14] _ On the structure of compact complex analytic surfaces, I, Amer. J. Math., 86 (1964), 751-798.

[15] Y. Miyaoka, On the Chern numbers of surfaces of general type, Invent. Math., 42 (1977), 225-237.

[16] W. Pohl, Differential geometry of higher order, Topology, 1 (1962), 169-211.

[17] I. R. Porteous, Simple Singularities of Maps, Proc. Liverpool Singularities, Springer Lecture Notes in Mathematics, vol. 192, 1971.

[18] C. Segre, Su una classe di superficie degl' iperspazî, legata colle equazioni lineari alle derivate parziali di $2^{0}$ ordine, Atti Acc Torini, 42 (1906-07), 1047-1079.

[19] T. Shifrin, The kinematic formula in complex integral geometry, Trans. Amer. Math. Soc., 264 (1981), 255-293.

[20] F. Sperenza, Le varietà le cui su erficie non ammettono spazi osculatori di dimensione regolare, Atti Sem. Mat. Fir. Univ. Modena, 17 (1968), 247-251.

[21] H.-S. Tai, On Frenet fames of complex submanifolds in complex projective space, preprint.

[22] E. Togliatti, Alcunî esempi di superficie algebriche degli iperspazî che rappersentano un' equazione di Laplace, Commentari Math. Helv., 1 (1929), 255-272.

Received January 7, 1985. Supported in part by a University of Georgia Faculty Research Grant.

UNIVERSITY OF GEORGIA

ATHENS, GA 30602 


\section{PACIFIC JOURNAL OF MATHEMATICS EDITORS}

V. S. VARADARAJAN

(Managing Editor)

University of California

Los Angeles, CA 90024

HERBERT CLEMENS

University of Utah

Salt Lake City, UT 84112

R. FINN

Stanford University

Stanford, CA 94305
HERMANN FLASCHKA

University of Arizona

Tucson, AZ 85721

RAMESH A. GANGOLLI

University of Washington

Seattle, WA 98195

VAUGHAN F. R. JONES

University of California

Berkeley, CA 94720

ROBION KIRBY

University of California

Berkeley, CA 94720
C. C. MOORE

University of California

Berkeley, CA 94720

H. SAMELSON

Stanford University

Stanford, CA 94305

HAROLD STARK

University of California, San Diego

La Jolla, CA 92093

\section{ASSOCIATE EDITORS}

R. ARENS

E. F. BECKENBACH

B. H. NEUMANN (1906-1982)
F. WOLF

K. YOSHIDA

\section{SUPPORTING INSTITUTIONS}

UNIVERSITY OF ARIZONA

UNIVERSITY OF BRITISH COLUMBIA

CALIFORNIA INSTITUTE OF TECHNOLOGY

UNIVERSITY OF CALIFORNIA

MONTANA STATE UNIVERSITY

UNIVERSITY OF NEVADA, RENO

NEW MEXICO STATE UNIVERSITY

OREGON STATE UNIVERSITY
UNIVERSITY OF OREGON UNIVERSITY OF SOUTHERN CALIFORNIA

STANFORD UNIVERSITY

UNIVERSITY OF HAWAII

UNIVERSITY OF TOKYO

UNIVERSITY OF UTAH

WASHINGTON STATE UNIVERSITY

UNIVERSITY OF WASHINGTON 


\section{Pacific Journal of Mathematics}

\section{Vol. 123, No. $1 \quad$ March, 1986}

Maria Emilia Alonso García, A note on orderings on algebraic varieties $\ldots \ldots 1$

F. S. De Blasi and Józef Myjak, On continuous approximations for

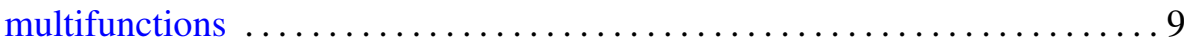

Frank Albert Farris, An intrinsic construction of Fefferman's CR metric . . . 33 Antonio Giambruno, P. Misso and Francisco César Polcino Milies, Derivations with invertible values in rings with involution $\ldots . \ldots . \ldots .47$

Dan Haran and Moshe Jarden, The absolute Galois group of a pseudo real

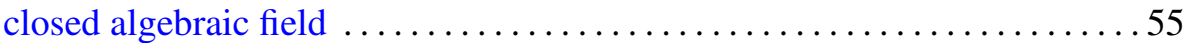

Telemachos E. Hatziafratis, Integral representation formulas on analytic

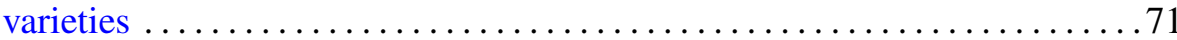

Douglas Austin Hensley, Dirichlet's theorem for the ring of polynomials

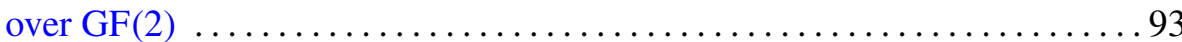

Sofia Kalpazidou, On a problem of Gauss-Kuzmin type for continued

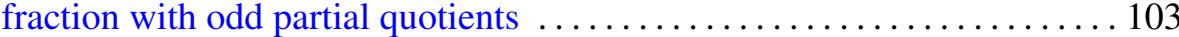

Harvey Bayard Keynes and Mahesh Nerurkar, Ergodicity in affine

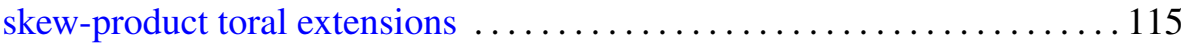

Thomas Landes, Normal structure and the sum-property $\ldots \ldots \ldots \ldots \ldots 127$

Anthony To-Ming Lau and Viktor Losert, Weak*-closed complemented invariant subspaces of $L_{\infty}(G)$ and amenable locally compact groups ...149 Andrew Lelek, Continua of constant distances in span theory . . . . . . . . 161 Dominikus Noll, Sums and products of $B_{r}$ spaces $\ldots \ldots \ldots \ldots \ldots \ldots \ldots \ldots$ Lucimar Nova, Fixed point theorems for some discontinuous operators 189

A. A. S. Perera and Donald Rayl Wilken, On extreme points and support points of the family of starlike functions of order $\alpha$

Massimo A. Picardello, Positive definite functions and $L^{p}$ convolution operators on amalgams ........................

Friedrich Roesler, Squarefree integers in nonlinear sequences ......... 223

Theodore Shifrin, The osculatory behavior of surfaces in $\mathbf{P}^{5}$ 